\title{
THEORY OF SINGLE BUNCH STABILITY AND DYNAMICS IN LINACS WITH STRONG WAKEFIELDS AND MISALIGNMENTS
}

\author{
G. Guignard and J. Hagel
}

The basic method we propose in order to solve analytically the equation of motion of a relativistic single-bunch travelling in a linac, in the presence of wakefields, has been summarized in a preceding report [1]. The extended treatment presented here includes the quadrupole transverse displacements, the chromatic variation of the magnetic focusing, the energy spread along the bunch and possible microwave quadrupoles. It deals with a Gaussian distribution of charge, linear variation of the wakefields within the bunch and smooth focusing. The energy is assumed to be constant in linac sectors, but increases from one sector to the next to simulate acceleration. The longitudinal and transverse equations of motion are solved, the second by using the perturbation method with partial expansions developed for this theory. The localized nature of the misalignment kicks and their superposition property are preserved by using thin lenses. The causality of the downstream oscillations due to these kicks is introduced via Heaviside functions. These ideas make it possible to build an analytical model for quadrupole misalignments and correlated displacements due to trajectory corrections. The resulting theory provides algebraic expressions for BNS damping, tune shifts, transverse off-sets and emittance dilution. It represents a significant break-through complementing the simulations and reproducing the oscillations observed numerically.

Submitted to Nuclear Instruments $\&$ Methods in Physics Research A

Geneva, Switzerland

16.02.1999

SL/Div Reps 


\section{Contents}

1 Introduction $\quad 3$

2 Equations of motion and simplification $\quad 4$

2.1 Introduction of Simplifications _ . . . . . . . . . . . . . . . 5

2.2 Separation of Variables in the transverse Equation $\ldots \ldots \ldots \ldots$

2.3 Weak focusing Approximation . . . . . . . . . . . . . . 8

3 Steps in solving the equations of motion $\quad 9$

3.1 Superposition Principle. . . . . . . . . . . . . . . . . . . 9

3.2 Causality Principle . . . . . . . . . . . . . . . . . . . . . 11

3.3 Partial Perturbation Expansion Method . . . . . . . . . . . . . . 12

4 Solution of the longitudinal equation of motion $\quad 13$

5 Solution of the transverse equation of motion $\quad 14$

5.1 The equation for the coherent oscillation $X(s) \ldots \ldots \ldots \ldots$

5.2 The equation for the incoherent oscillation $y(s, z) \ldots \ldots \ldots$

6 Trajectory correction and time-dependent misalignment 18

6.1 Quadrupole random misalignments . . . . . . . . . . . . . . . 18

6.2 Quadrupole displacements after trajectory correction . . . . . . . . . . . . 19

6.2 .1 Balistic correction . . . . . . . . . . . . . . . 19

6.2 .2 One-by-one trajectory correction . . . . . . . . . . . . . . 20

6.2.3 Quadrupole drifts due to ground motion . . . . . . . . . . . . . . 21

7 Comparison of the analytical results to MUSTAFA 22

8 Conclusions $\quad 25$

$\begin{array}{lll}\text { A Solution of the betatron equation with dipole kicks } & 27\end{array}$

B Properties and approximation of the Heaviside function $\quad 29$

C The partial perturbation expansion method 32

D The logistic map and random number generation $\quad 34$ 


\section{Introduction}

The present analysis deals with solving the equations of motion for individual, relativistic particles distributed in a single bunch, travelling along a linac with unavoidable wakefields and quadrupole misalignments. This analytical treatment of the beam dynamics is a very useful complement to the numerical simulations we have done with our tracking code MUSTAFA [2]. It also provides explicit solutions of the beam motion for our better understanding of the physics involved and of the dependence on the various parameters. Using linac and beam models as realistic as possible, it gives closed expressions for critical quantities such as the tune shift in the bunch, the transverse bunch off-sets, the energy distribution within the bunch and the emittance dilution, helping to formulate scaling laws. Last but not least, this theory opens the way to the creation of a simulation algorithm based on the explicit analytical solutions, using the fact that the wake-field effects are integrated over the bunch and therefore speed-up the simulations. This algorithm presently in preparation will be added into MUSTAFA for comparing its results with the fully numerical simulations.

We address the mostly interesting question of the effects of wakefields and misalignments on the transverse emittance, starting from the equation of motion that describes the interaction of the bunch tail with the wakefield generated by the head as well as the bunch oscillations under the impact of the misalignments and/or displacements of the focusing quadrupoles (Section 2). This may lead to beam break-up instabilities and emittance growth ([3] to [6]), counteracted by BNS damping and autophasing ([7] to [10]). Attempts to describe and estimate the emittance dilution and preservation have been done in a recent past ([11] to [15]). They however rely on severe simplifications and on a standard perturbation expansion which generates at each order artificial resonant terms called secular terms that only cancel each other when the expansion goes to infinity.

In order to prevent the mentioned difficulty with the standard perturbation, we developed a specific method to prevent the appearance of artificial secular (resonant) terms by keeping at any order of the perturbation expansion the intrinsic tune-shift that tends to stabilize the motion. This approach, we call 'the partial perturbation expansion method', is described in Section 3 and Appendix C. Other important steps have been purposedly taken in solving the equations of motion, based on the superposition and the causality principles (Section 3, Appendices A and B), in order to allow an accurate description of the local kicks related to the transverse displacements of the quadrupoles and their effects on the betatron oscillations. These were key elements of the theory for making the analytical integration of the wakefield -related integrals possible through the whole mathematical development.

The obtained solutions for the longitudinal and transverse equations of motion are given in Sections 4 and 5, respectively, with the hint of how to deduce from the center-of-gravity off-sets along the bunch, the contribution to the emittance which (quadratically) add to the intrinsic transverse emittance imposed at injection. An additional interesting feature of this theory stands in the final form of the incoherent off-sets within the bunch, that contains a sum over the quadrupole displacements $x_{k}$. This allows to predict the effects of correlated or uncorrelated quadrupole displacements provided there is a function or a recurrence relation that defines the $x_{k}$. Section 6 describes the possibility to include in our model the random displacements of the quadrupoles (analytically generated by using the logistic map properties, as shown in Appendix D), their shifts required for trajectory corrections and their drifts associated to ground motion.

Results from the theory concerning the center-of-gravity off-sets that take place within a single bunch and contribute to the emittance growth when the bunch travels down the linac are given in Section 7. They are compared in similar conditions with the numerical tracking results obtained with MUSTAFA and it is shown that the agreement between the two is good. 


\section{Equations of motion and simplification}

Since in most linear colliders a flat beam design (low vertical to horizontal beam size ratio) is used, emittance blow up due to wakefields is most critical in the vertical plane and leads to serious reductions in luminosity at interaction when no correction is applied. The equations of motion in the longitudinal and vertical plane, in the presence of longitudinal and transverse wakefields and in the relativistic limit, are [1] [17]:

$$
\begin{aligned}
& \frac{\partial \gamma}{\partial s}=\frac{e U}{m_{0} c^{2}} \cos \left(k_{R F} z-\bar{\Phi}_{R F}\right)-C \int_{0}^{z} \rho\left(z^{*}\right) W_{L}\left(z-z^{*}\right) d z^{*} \\
& \frac{\partial^{2} x(s, z)}{\partial s^{2}}+\frac{1}{\gamma(s, z)} \frac{\partial \gamma(s, z)}{\partial s} \frac{\partial x(s, z)}{\partial s}-K(s)[1+\Delta k(s, z)] x(s, z)= \\
& \frac{C}{\gamma(s, z)} \int_{0}^{z} \rho\left(z^{*}\right) W_{T}\left(z-z^{*}\right) x\left(s, z^{*}\right) d z^{*}+ \\
& \frac{C}{\gamma(s, z)} x_{A}(s) \int_{0}^{z} \rho\left(z^{*}\right) W_{T}\left(z-z^{*}\right) d z^{*}+K(s)[1+\Delta k(s, z)] x_{Q}(s)
\end{aligned}
$$

with

$$
\Delta k(s, z)=-\delta(s, z)+\alpha_{0} \sin \left(k_{R F} z+\bar{\Phi}_{R F Q}\right)
$$

where

$$
\delta(s, z)=\frac{\gamma(s, z)-\gamma(s, 0)}{\gamma(s, z)}
$$

The quantities contained in these two semi coupled equations have the following meanings:

- $s, z[m]$ : The independent variables $s$ and $z$ describe the position of the head of the bunch inside the linac and the position inside the bunch. The position $z=0$ represents the head of the bunch and $z=l_{B}>0$ the tail of the (truncated) bunch. In this report we shall use a bunch truncated at $\pm 2 \sigma_{z}$ (hence $l_{B}=4 \sigma_{z}$ ), where $\sigma_{z}[m]$ represents one standard deviation for a Gaussian bunch.

- $\underline{\gamma(s, z):}$ The energy Lorentz factor within the bunch and at some position $s$ inside the linac.

- $\underline{e[A s]:}$ The elementary charge.

- $\underline{U}[V]$ : The accelerating field peak voltage.

- $m_{0} c^{2}[A V s]$ : The rest energy of the electron.

- $\underline{k}_{R F}\left[m^{-1}\right]$ : The RF wave number.

- $\bar{\Phi}_{R F}$ : The RF phase of the head of the bunch. It is related to the phase at the bunch center by

$$
\bar{\Phi}_{R F}=\Phi_{R F}+\frac{l_{B} \pi}{\lambda_{R F}}
$$

- $C\left[\frac{A s}{V}\right]:$ A constant (independent of $s$ and $z$ ) defined as

$$
C=4 \pi \epsilon_{0} r_{e} N
$$

where $\epsilon_{0}$ is the dielectric constant of the vacuum, $r_{e}$ the classical electron radius and $N$ the number of charged particles in the electron or positron bunch. 
- $\rho(z)\left[m^{-1}\right]$ : The normalized line density charge distribution of the bunch. The distribution is assumed to be Gaussian and truncated at the head and the tail of the bunch.

- $W_{L}(z)\left[\frac{V}{A s m}\right]:$ The longitudinal wakefield along the bunch.

- $x(s, z)[m]:$ The vertical transverse betatron coordinate.

- $K(s)\left[m^{-2}\right]:$ The magnetic focusing quadrupole strength. In our cas we use a thin lens FODO lattice, i.e.

$$
K(s)=\frac{2}{L} \sin \frac{\mu}{2} \sum_{k=1}^{N_{Q}}(-1)^{k} \delta(s-k L)
$$

where $k$ is the quadrupole index, $L$ is the distance between successive quadrupoles and $\mu$ the phase advance per FODO cell.

- $\Delta k(s, z)$ : An $s$ and $z$ dependent correction to the quadrupole focusing given in Eq. (3) which contains the energy spread along the bunch as function of $s$ defined in Eq. (4) as well as RF quadrupoles with strength $\alpha_{0}$. The phase of the RF quadrupoles $\bar{\Phi}_{R F Q}$ is given for $z=0$ and is related to the phase of the bunch center as

$$
\bar{\Phi}_{R F Q}=\Phi_{R F Q}+\frac{l_{B} \pi}{\lambda_{R F}}
$$

- $W_{T}(z)\left[\frac{V}{A s m^{2}}\right]:$ The transverse wakefield along the bunch.

- $x_{A}(s)[m]$ : The missalignments of the RF cavities.

- $x_{Q}(s)[m]:$ The missalignments of the magnetic focusing quadrupoles.

\subsection{Introduction of Simplifications}

While it is straightforward to solve the longitudinal equation (1) in its given form by a simple quadrature, the transverse coordinate $x$ follows a linear, second order, partial and inhomogeneous integro-differential equation (2) in two independent variables $s$ and $z$ and with nonconstant coefficients. A closed form solution procedure to this equation is not known so far and therefore it is indicated to apply some simplifications in order to facilitate an approximate analytic treatment. These modifications have to be chosen with care to not loose or modify strongly the essential dynamical properties of this complex system. In this paper we introduce the following simplifications:

1. The missalignments of the RF cavities $x_{A}(s)$ are neglected since there effect on the dynamics is much weaker then the one of the quadrupole missalignments. This is because $x_{A}(s)$ is multiplied by the transverse wakefield which is considered as a perturbation to the unperturbed betatron motion.

2. The Lorentzfactor $\gamma(s, z)$ in Eq. (2) is considered constant along $s$ and $z$ and set equal to the injected nominal $\gamma(0,0)=\gamma_{0}$ in each linac sector. Hence the acceleration term proportional to $\frac{\partial x}{\partial s}$ on the left hand side of Eq. (2) vanishes and the function $\gamma(s, z)$ on the right hand side of the equation is replaced by the constant $\gamma_{0}$. This is mainly justified by the fact that neglecting the damping term leads even to slightly pessimistic orbit results, since due to the damping a factor $\gamma(s, z)^{-1 / 2}$ is created and in addition the first integral driving term on the right hand side has a constant factor $1 / \gamma_{0}$ instead of a function decreasing proportional to $\frac{1}{s}$ within the given linac sector. 
3. The contribution $\delta(s, z)$ in Eq. (3) is replaced by its asymptotic value $\delta(z)=\lim _{s \rightarrow \infty} \delta(s, z)$..

4. As in [1] the actual Gaussian charge distribution along the truncated bunch is replaced by its fourth order Chebychev approximation in this interval which is given by

$$
e^{-\frac{1}{2} x^{2}} \approx 1-\frac{41}{100} x^{2}+\frac{1}{20} x^{4}
$$

and this approximation agrees with the truncated Gaussian to less than $4 \%$ of the maximum within the entire $x$ interval. Using this approximation we avoid the appearence of complicated Errorfunctions in the process of quadratures w.r.t the $z$ coordinate. After normalization $\rho(z)$ then reads:

$$
\rho(z)=\frac{75}{46 l_{B}}\left[\frac{1}{20}\left(\frac{4 z}{l_{B}}-2\right)^{4}+\frac{41}{100}\left(\frac{4 z}{l_{B}}-2\right)^{2}+1\right]
$$

A comparison between the truncated Gaussian distribution and its Chebyshev approximation can be seen in Fig. 1

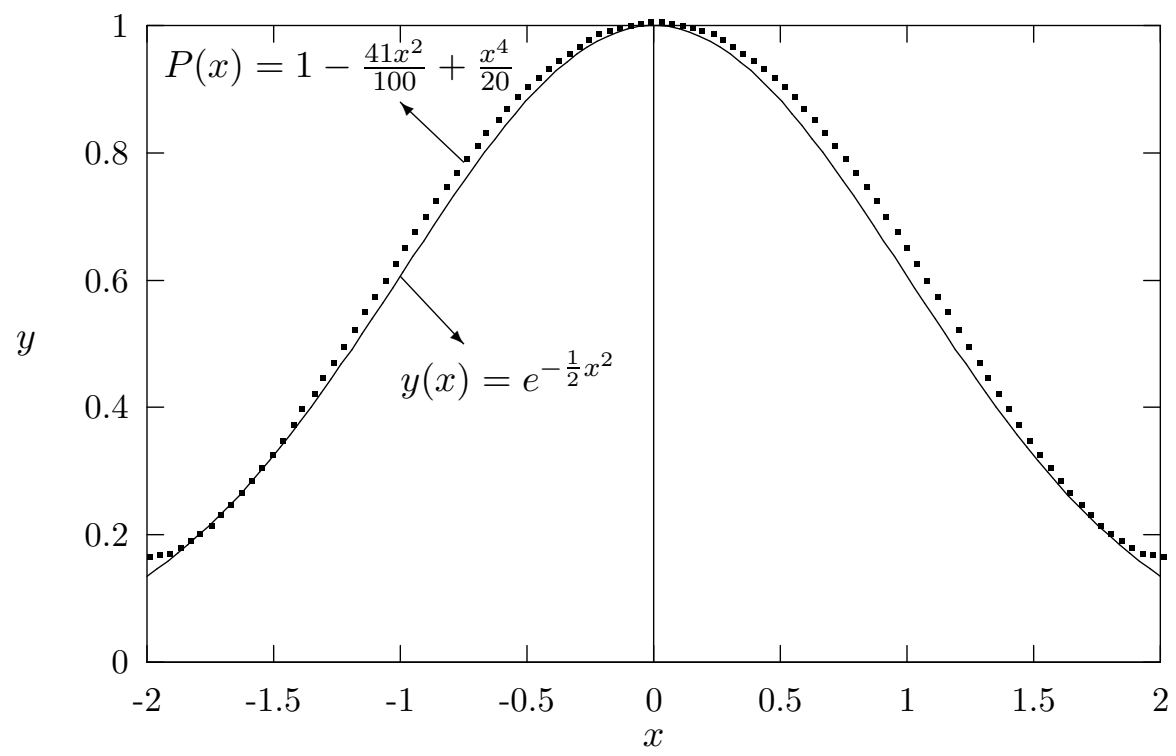

Fig. 1: Comparison between Gaussian and polynomial approximation

5. The longitudinal and transverse wakefields along the bunch are modeled by linear functions as

$$
W_{T}(z)=\frac{W_{T 0}}{l_{B}} z
$$

and

$$
W_{L}(z)=W_{L 0}+\frac{W_{L 1}-W_{L 0}}{l_{B}} z
$$

where $W_{T 0}$ is the value of the transverse wakefield at the tail of the bunch and $W_{L 0}$ and $W_{L 1}$ are the values of the longitudinal wakefield at the head and tail of the bunch respectivly. 
With all these simplifications, the equations of motion (1) and (2) read as

$$
\begin{gathered}
\frac{\partial \gamma}{\partial s}=\frac{e U}{m_{0} c^{2}} \cos \left(k_{R F} z-\bar{\Phi}_{R F}\right)-C \int_{0}^{z} \rho\left(z^{*}\right)\left[W_{L 0}+\frac{W_{L 1}-W_{L 0}}{l_{B}}\left(z-z^{*}\right)\right] d z^{*} \\
\frac{\partial^{2} x}{\partial s^{2}}-K(s)[1+\Delta k(z)] x=\frac{W_{T 0} C}{\gamma_{0}} \int_{0}^{z} \rho\left(z^{*}\right)\left(z-z^{*}\right) x\left(s, z^{*}\right) d z^{*}+K(s)[1+\Delta k(z)] x_{Q}(s)
\end{gathered}
$$

and the initial conditions are

$$
\begin{aligned}
& \gamma(0, z)=\gamma_{0} \\
& x(0, z)=0 \\
& \frac{d x}{d s}(0, z)=0
\end{aligned}
$$

While in [1] we deal with an injection offset in the form

$$
\begin{aligned}
& x(0, z)=\alpha_{0} \\
& \frac{d x}{d 0}(0, z)=\alpha_{1} z
\end{aligned}
$$

in order to simulate the existance of one missaligned focusing element, we do not do so in the present work but rather deal with a real case of distributed missaligned quadrupoles along the linac represented by the function $x_{Q}(s)$.

\subsection{Separation of Variables in the transverse Equation}

Frequently the analytic evaluation of the solution of a partial differential or integro-differential equation becomes easier if one applies a partial or total separation of the independent variables. In our case a partial separation proved to be the most favorite one and we write the total solution of the transverse equation of motion as

$$
x(s, z)=X(s)+y(s, z)
$$

Then we obtain two new equations for $X$ and $y$ as

$$
\begin{aligned}
& \frac{d^{2} X(s)}{d s^{2}}-K(s) X(s)=K(s) x_{Q}(s) \\
& \frac{\partial^{2} y(s, z)}{\partial s^{2}}-K(s)[1+\Delta k(z)] y(s, z)=X(s)\left[K(s) \Delta k(z)+\frac{C W_{T 0}}{\gamma_{0} l_{B}} \int_{0}^{z} \rho\left(z^{*}\right)\left(z-z^{*}\right) d z^{*}\right]+ \\
& \frac{C W_{T 0}}{\gamma_{0} l_{B}} \int_{0}^{z} \rho\left(z^{*}\right)\left(z-z^{*}\right) y\left(s, z^{*}\right) d z^{*}+K(s) \Delta k(z) x_{Q}(s)
\end{aligned}
$$

with initial conditions

$$
\begin{aligned}
& X(0)=\frac{d x}{d s}(0)=0 \\
& y(0, z)=\frac{d y}{d s}(0, z)=0
\end{aligned}
$$




\subsection{Weak focusing Approximation}

In order to simplify the subsequent analysis, we apply the well known weak focusing approximation. This consists of considering the general solution of Hills equation

$$
\frac{d^{2} x}{d s^{2}}-K(s) x=0
$$

in the form

$$
x(s)=\sqrt{\beta(s)} \cos \left[\int_{0}^{s} \frac{d s^{*}}{\beta\left(s^{*}\right)}+\Phi\right]
$$

and replacing the periodic beta function $\beta(s)$ by its zero order Fourier component,

$$
\beta(s)=\frac{1}{2 L} \int_{0}^{2 L} \beta(s) d s=\bar{\beta}
$$

Hence $x(s)$ becomes

$$
x(s)=A \cos \left[\frac{s}{\bar{\beta}}+\Phi\right]=A \cos (q s+\Phi)
$$

where $q=1 / \bar{\beta}$ is the socalled weak focusing tune of the lattice under consideration.

However in our case we apply this approach in a selective way. This means that we apply the weak approximation in Eqs. (21) and (22) only to terms not being coefficients of the function $x_{Q}(s)$ describing the quadrupole missalignments. The reason for this selective approach is that we would like to preserve the localized nature of the magnetic missalignments along the linac which is essential for obtaining the correct description of the resulting emittance dilution due to missalignments and wakefields. In addition this preservation of the localized nature opens an easy way to describe any of the correction schemes in use in a straightforward analytical way as will be shown in Section 6. Applying this philosophy the equation for $X(s)$ becomes:

$$
\frac{d^{2} X}{d s^{2}}+q^{2} X=K(s) x_{Q}(s)
$$

where $q$ is the weak focusing tune calculated by the inverse average beta function of an on energy FODO lattice [17]

$$
q=\frac{1}{\bar{\beta}}=\left[L\left(\cot \frac{\mu_{\text {cell }}}{2}+\frac{2}{3} \tan \frac{\mu_{\text {cell }}}{2}\right)\right]^{-1}
$$

In the case of Eq. (22) the weak focusing tune $\bar{q}$ is given by the inverse average of the beta function of an off energy FODO lattice. Starting with the homogeneous equation

$$
\frac{\partial^{2} y}{\partial s^{2}}-K(s)[1+\Delta k(z)] y=0
$$

we construct the transfermatrix $M(s)$ over one FODO period (transfering the solution vector $\left(y, y^{\prime}\right)$ from the position $s$ to $\left.s+P, P=2 L\right)$ by multiplication of five partial matrices:

$$
M(s)=D(s) M_{F} D(P / 2) M_{D} D(P / 2-s)
$$

where

$$
\begin{gathered}
D(P / 2-s)=\left(\begin{array}{cc}
1 & P / 2-s \\
0 & 1
\end{array}\right) \cdots \text { drift from s to QD } \\
M_{D}=\left(\begin{array}{cc}
1 & 0 \\
\frac{4}{P} \sin \frac{\mu}{2}[1+\Delta k(z)] & 1
\end{array}\right) \cdots \text { horizontally defocusing matrix for QD }
\end{gathered}
$$




$$
\begin{gathered}
D(P / 2)=\left(\begin{array}{cc}
1 & P / 2 \\
0 & 1
\end{array}\right) \cdots \text { drift over half a period } \\
M_{D}=\left(\begin{array}{cc}
1 & 0 \\
-\frac{4}{P} \sin \frac{\mu}{2}[1+\Delta k(z)] & 1
\end{array}\right) \cdots \text { horizontally focusing matrix for QF } \\
D(s)=\left(\begin{array}{ll}
1 & s \\
0 & 1
\end{array}\right) \cdots \text { drift from QF to } \mathrm{s}
\end{gathered}
$$

Then $\beta(s)$ is computed in the usual way Ref. [FODO] by:

$$
\beta(s)=\frac{M_{12}}{\sin \mu}=\frac{M_{12}}{\sqrt{1-\cos ^{2} \mu}}=\frac{M_{12}}{\sqrt{1-\frac{1}{4} \operatorname{Tr}^{2}(M)}}
$$

As well known, the function $\beta(s)$ turns out to be a quadratic polynomial in $s$ between two quadrupoles. The weak focusing tune is then computed in the usual way and is:

$$
\bar{q}(z)=\frac{3(1+\Delta k(z))}{L\left(3-(1+\Delta k(z))^{2} \sin ^{2} \frac{\mu}{2}\right)} \sqrt{(1-\cos \mu)\left[2-(1+\Delta k(z))^{2}(1-\cos \mu)\right]}
$$

and $\Delta k(z)$ is given by Eq. (3). The partially tedious algebra involved in computing $\bar{q}(z)$ has been performed by use of the MAPLE program [16].

The term $K(s) \Delta k(z)$ in the coefficient of $X(S)$ in Eq. (22) can be treated in the following way:

$$
K(s) \Delta k(z)=K(s)(1+\Delta k(z))-K(s) \Longrightarrow \bar{q}(z)^{2}-q^{2}
$$

Hence the new equations for $X$ and $y$ with the weak focusing approximation applied to all terms not multiplying $x_{Q}(s)$ becomes

$$
\begin{aligned}
& \frac{d^{2} X}{d s^{2}}+q^{2} X=K(s) x_{Q}(s) \\
& \frac{\partial^{2} y}{\partial s^{2}}+\bar{q}^{2}(z) y=X(s)\left[\frac{C W_{T 0}}{l_{B} \gamma_{0}} \int_{0}^{z} \rho\left(z^{*}\right)\left(z-z^{*}\right) d z^{*}-\left(q^{2}-\bar{q}^{2}(z)\right)\right] \\
& +\frac{C W_{0}}{\gamma_{0} l_{B}} \int_{0}^{z} \rho\left(z^{*}\right)\left(z-z^{*}\right) y\left(s, z^{*}\right) d z^{*}+K(s) \Delta k(z) x_{Q}(s)
\end{aligned}
$$

\section{$3 \quad$ Steps in solving the equations of motion}

\subsection{Superposition Principle.}

Each single misalignment of the quadrupoles generates a variation of the orbit and a downstream oscillation of the beam with the wave number $q$. Hence, when the bunch travels in the linac and passes one more quadrupole, it is submitted to an additional "kick", the effect of which adds to those of all the preceding "kicks". In this section, we like to discuss more precisely the superposition of all these kick effects, restarting from the equation (29) of the betatron motion.

If the quadrupole is infinitely thin, its misalignment creates a single localised kick and if the quadrupole has a finite length, the effect of its misalignment results from integrating a continuous series of kicks. In both cases, the solution of the Eq. (41) will result from the response of a single localised unity-kick, also named the Green function. The Green function is solution of the following equation: 


$$
\frac{\partial^{2} G\left(s, s_{k}\right)}{\partial s^{2}}+q^{2} G\left(s, s_{k}\right)=\delta\left(s-s_{k}\right)
$$

with the following initial conditions

$$
\begin{aligned}
& G\left(s<s_{k}\right)=0 \\
& G\left(s=s_{k}\right)=0 \\
& G^{\prime}\left(s=s_{k}\right)=1 .
\end{aligned}
$$

For this unity kick at $s=s_{k}$, the Green function can be written as:

$$
G\left(s, s_{k}\right)=\frac{1}{q} \sin \left[q\left(s-s_{k}\right)\right] H\left(s-s_{k}\right)
$$

where $H\left(s-s_{k}\right)$ is the Heaviside function or step-function.

More generally we can write for distributed unity-kicks at variable positions $s^{*}$ that:

$$
G\left(s, s^{*}\right)=\frac{1}{q} \sin \left[q\left(s-s^{*}\right)\right] H\left(s-s^{*}\right)
$$

and it can be demonstrated (Appendix A) that, integrating over a multiple of the focusing period $\lambda_{\beta}$, the following expression is solution of Eq. (41):

$$
X(s)=\int_{0}^{n \lambda_{\beta}} G\left(s, s^{*}\right) \theta\left(s^{*}\right) d s^{*}
$$

The function $\theta\left(s^{*}\right)$ represents the amplitude of the distributed kicks due to quadrupole misalignments over the $n$ focusing periods considered. This integral form is the only one strictly valid for continuous series of kicks, e.g. for quadrupoles with finite length, since over this length the amplitude of the trajectory changes while the kicks act.

Let us now consider a succession of isolated kicks or misalignments associated with thin lenses. Each kick amplitude can be written as :

$$
\theta_{k}=K\left(s_{k}\right) l_{k} x_{k}=(-1)^{k} x_{k} \frac{2}{L} \sin \left(\frac{\mu}{2}\right)
$$

where $K\left(s_{k}\right)$ and $l_{k}$ are the strength and length of the quadrupole $k$ respectively, L the distance separating two quadrupoles of the lattice, $\mu$ the phase advance per cell and $x_{k}$ the transverse displacement of the magnetic lens considered. The change of sign in the front of tis equation corresponds to the alternate focusing of the FODO lattice.

For a succession of local kicks, the function $\theta\left(s^{*}\right)$ in Eq. (47) has to be replaced by a sum of Dirac functions with kick amplitude $\theta_{k}$, as follows

$$
X(s)=\int_{0}^{n \lambda_{\beta}} G\left(s, s^{*}\right) \sum_{k} \theta_{k} \delta\left(s^{*}-s_{k}\right) d s^{*}
$$
holds

Replacing $\theta_{k}$ and $G\left(s, s^{*}\right)$ by their expressions (48) and (46), the next expression of $X(s)$

$$
X(s)=\frac{2}{q L} \sin \left(\frac{\mu}{2}\right) \int_{0}^{n \lambda \beta} \sum_{k}(-1)^{k} x_{k} \delta\left(s^{*}-s_{k}\right) \sin \left[q\left(s-s^{*}\right)\right] H\left(s-s^{*}\right) d s^{*}
$$


and can be written after integration (with the Dirac function)

$$
X(s)=\frac{2}{q L} \sin \frac{\mu}{2} \sum_{k}(-1)^{k} x_{k} \quad \sin \left[q\left(s-s_{k}\right)\right] \quad H\left(s-s_{k}\right)
$$

The last relation is the formal expression of the superposition principle of the quadrupole misalignment effects. The performed integral shows that it only applies if the motion is periodic (with a constant wave number q), the kicks are local (thin lenses represented by Dirac functions) and a multiple of full focusing periods is considered. With a constant distributed focusing of wave number $q$ in the homogeneous part of Eqs. (41) and (42) and localized kicks associated with thin quadrupole misalignments in their inhomogeneous parts, the superposition principle described above is fully valid and will be used subsequently.

\subsection{Causality Principle}

The causality simply expresses the fact that the effects of some excitation can only follow, in time and coordinate, the instant and position of the excitation source. We obviously have to apply it in particular to the trajectory oscillations that are provoked by the displacement $x_{Q}$ of a single quadrupole. As explained in the preceding section, it is always possible to decompose the effect of this displacement in a series of angular kicks and consider the effect of a single kick for the discussion of the causality. In this particular application, the effect is given by the Green function solution of Eq. (43) with the initial conditions (44). The causality is mathematically described by the condition that the trajectory is strictly equal to zero for $s<s_{k}$, experiences a unity angular-deviation at $s=s_{k}$ and zero amplitude, and finally oscillates with a wave-number q when $s>s_{k}$ as

$$
\frac{1}{q} \sin \left[q\left(s-s_{k}\right)\right] \quad s>s_{k}
$$

The simplest way to write a single expression consists of using the step function $H\left(s-s_{k}\right)$, called the Heaviside function, which takes the value 0 when $s<s_{k}$ and 1 when $s>s_{k}$.

$$
\begin{array}{lll}
H\left(s-s_{k}\right)=0 & \text { for } & s \leq s_{k} \\
H\left(s-s_{k}\right)=1 & \text { for } & s>s_{k}
\end{array}
$$

Multiplying the oscillating function (52) by the Heaviside function, as done in the Section 3.1, provides the expression which includes the causality principle and can easily be carried over the integrals of the motion. As shown below, the solution of the equation of motion will contain a sum over the quadrupoles of functions multiplied by Heaviside's functions at $s-s_{k}$.

This solution contains both the quadrupole coordinates $s_{k}$ and the variable $s$. It could be convenient to eventually separate these two quantities in order to collect in front of the algebraic expressions obtained after integration of the motion equation all the terms depending on the quadrupole index $k$ (including $s_{k}$ ); hence the sum of the quadrupole contributions to the bunch off-sets would be done initially and independently of the calculation of the s-dependence of the motion.

This is possible if the functions involved can be replaced by products of functions of $s$ with functions of $s_{k}$. Since sinusoidal functions obviously have the required property, a development in Fourier series of the function $H$ is the logical thing to do, so as

$$
H(s)=\sum_{m=1}^{\infty} h_{m} \sin \left(\omega_{m} s\right)
$$


The Appendix B shows how this is achieved and gives the form of the quantities $h_{m}$ and $\omega_{m}$

$$
\begin{array}{ll}
h_{0}=\frac{1}{2} & h_{m}=\frac{2}{\pi(2 m-1)} \\
\omega_{0}=0 & \omega_{m}=\frac{(2 m-1) \pi}{L_{S}}
\end{array}
$$

where $L_{S}$ is the total length of a linac sector.

In addition, when the perturbation of the equation of motion in the presence of displacements $x_{Q}(s)$ is dealt with (Section 5), this setting in evidence of the terms depending on $k$ remains only possible if the Heaviside function is extracted from the integral which is performed to obtain the perturbed solution $y(s, z)$. This can indeed be done by using the following identity

$$
\int_{0}^{s} f\left(s^{*}\right) H\left(s^{*}-s_{k}\right) d s^{*}=H\left(s-s_{k}\right) \int_{s_{k}}^{s} f\left(s^{*}\right) d s^{*}
$$

This interesting and fundamental relation is demonstrated in Appendix B and will be used when solving the equation for the incoherent part $y(s, z)$ of the motion.

\subsection{Partial Perturbation Expansion Method}

The idea consists of separating the perturbation of the solvable part of the equation or detuning from the perturbing driving term that contains the transverse wakefield.

Starting from the equation (42) for the incoherent part of the motion $y(s, z)$, the perturbation associated with detuning is clearly included in the expression of $\bar{q}(z)(39)$, that depends on the correction to the quadrupole focusing $\Delta k(s, z)$ given in Eq. (3). Let us mark this perturbation with the symbol $E$ which recalls that the detuning is small, $E$ being replaced by one at the end of the perturbation treatment. By contrast, the perturbing driving term is contained in the integral over $z$ of the transverse wakefield multiplied by the charge density and the incoherent off-set $y\left(s, z^{*}\right)$ in the bunch. We shall mark the driving term by the usual symbol $\epsilon$ which also means that the perturbation is small and which is also eventually replaced by 1 . This notation precisely separates the detuning perturbation from the driving-term perturbation, as advocated and as indicated in the following equation

$$
\begin{aligned}
& \frac{\partial^{2} y}{\partial s^{2}}+\bar{q}^{2}[\mathbf{E} \Delta k(s, z)] y= \\
& {\left[\frac{C}{\gamma_{0}} \int_{0}^{z} \rho\left(z^{*}\right) w^{T}\left(z-z^{*}\right) d z^{*}-\left[q^{2}-\bar{q}^{2}(z)\right]\right] X(s)} \\
& +\varepsilon \frac{C}{\gamma_{0}} \int_{0}^{z} \rho\left(z^{*}\right) w^{T}\left(z-z^{*}\right) y\left(s, z^{*}\right) d z^{*} \\
& +k(s)\left[-\delta(z)+\Delta k_{R F Q}(s, z)\right] x_{Q}(s)
\end{aligned}
$$

The next step consists of making a perturbation expansion with respect to $\epsilon$ only, keeping the detuning of $q$ marked with $E$ as part of the unperturbed linear operator describing the harmonic oscillations. Not expanding the perturbation with respect to $\mathbf{E}$ preserves at all steps the full detuning of the linear motion, and prevents the rise of artificial resonant or secular terms.

At zero order, putting $\epsilon=0$ in the above equation for $y$, the solution called $y_{0}(E)$ can be worked out while including the driving term due to the coherent betatron motion $X(s)$ as well as the detuned wave-number $\bar{q}$. At first order in $\epsilon, y_{0}$ is then included in the integral of the right hand side and the next approximation $y_{1}(E)$ is deduced. Order by order, this procedure can 
be repeated, giving a series of contributions $y_{n}(E)$ which provides an approximation to within $O\left(\epsilon^{n+1}\right)$ of the exact solution ,

$$
y(s, z)=\sum_{n=0}^{N} y_{n}(E) \epsilon^{n}+O\left(\epsilon^{n+1}\right)
$$

This approach we called partial expansion method is more rigorously described in Appendix B, where it is compared to the standard Taylor expansion. It is proved to order two that the two methods provide exactly the same series when $E=\epsilon$ is substituted and the functions of $E$ are also expanded at the end of the partial expansion procedure; the remainders are however different. This result is then used to establish the conjecture that the partial expansion (59) agrees, for $E=\epsilon$, with the Taylor standard expansion, to any order N.

\section{Solution of the longitudinal equation of motion}

The equation for the Lorentzfactor $\gamma(s, z)$ given in (1) is solved in a straightforward way by integrationg the equation w.r.t. the independent variable $s$. This results in

$$
\gamma(s, z)=\gamma_{0}+\frac{e U s}{m_{0} c^{2}} \cos \left(k_{R F} z-\overline{P h} i_{R F}\right)-4 \pi \epsilon_{0} r_{e} N s R(z)
$$

with

$$
R(z)=\int_{0}^{z} W_{L}\left(z-z^{*}\right) \rho\left(z^{*}\right) d z^{*}
$$

The function $R(z)$ can be easily found by using the linear wakefield model of the longitudinal field inside a single bunch given by (12) and by using the definition

$$
\zeta=\frac{z}{l_{B}}
$$

as well as the Chebyshev model for the gaussian distribution within $-2 \sigma_{z} \leq z \leq+2 \sigma_{z}$ given by In this way, $R(z)$ is given by (10).

$$
\begin{aligned}
& R(z)=W_{L 0}\left(-\frac{16}{23} \zeta^{6}+\frac{144}{23} \zeta^{5}-\frac{559}{46} \zeta^{4}+\frac{157}{23} \zeta^{3}+\frac{6}{23} \zeta\right) \\
& +W_{L 1}\left(\frac{16}{23} \zeta^{6}-\frac{48}{23} \zeta^{5}+\frac{79}{46} \zeta^{4}+\frac{1}{23} \zeta^{3}+\frac{3}{23} \zeta^{2}\right)
\end{aligned}
$$

As explained above, the $W_{L 0}$ and $W_{L 1}$ are the values of the longitudinal wakefield at the head $(z=0)$ and tail $\left(z=l_{B}\right)$ of the gaussian bunch. The energy spread inside the bunch is defined by

$$
\delta(s, z)=\frac{\gamma(s, z)-\gamma(s, 0)}{\gamma(s, z)}
$$

As discussed above, however we instead use the asymptotic value of $\delta(s, z)$ as $s$ tends to infinity, hence we replace

$$
\delta(s, z) \rightarrow \delta(z)=\lim _{s \rightarrow \infty} \delta(s, z)
$$

The following table shows a comparison between the analytical and tracking results for $\sigma_{E}$ as function of $\Phi_{R F}$. The definition of $\sigma_{E}$ is:

$$
\sigma_{E}=\sqrt{\int_{0}^{z} \rho(z)(\delta(z)-\bar{\delta})^{2} d z}
$$


with

$$
\bar{\delta}=\int_{0}^{z} \rho(z) \delta(z) d z
$$

For this comparison we used $N=6 \cdot 10^{9}$ particles per bunch and $\sigma_{z}=0.2 \mathrm{~mm}$

\begin{tabular}{|c|c|c|}
\hline$\Phi_{R F}^{[o]}$ & $\sigma_{E}[\%]_{\text {analyt. }}$ & $\sigma_{E}[\%]_{\text {track }}$ \\
\hline 7 & 0.92 & 0.87 \\
\hline 8 & 0.81 & 0.77 \\
\hline 9 & 0.73 & 0.70 \\
\hline 10 & 0.69 & 0.66 \\
\hline 10.5 & 0.69 & 0.66 \\
\hline 11 & 0.70 & 0.67 \\
\hline 11.5 & 0.72 & 0.68 \\
\hline 12 & 0.75 & 0.71 \\
\hline 13 & 0.83 & 0.78 \\
\hline 14 & 0.94 & 0.89 \\
\hline 15 & 1.07 & 1.00 \\
\hline 16 & 1.21 & 1.14 \\
\hline 17 & 1.35 & 1.28 \\
\hline 18 & 1.50 & 1.43 \\
\hline 19 & 1.66 & 1.58 \\
\hline 20 & 1.82 & 1.74 \\
\hline
\end{tabular}

Table 1: Comparison between analysis and tracking for the energy spread

\section{Solution of the transverse equation of motion}

We now turn to the actual solution procedure of the separated equations (41) for $X(s)$ and (42) for $y(s, z)$ describing the transverse (vertical) motion of the single bunch.

\subsection{The equation for the coherent oscillation $X(s)$}

The equation (41) for the pure quadrupole lattice with misplaced quadrupoles has already been solved in Section 3.1 and $X$ turned out to be

$$
X(s)=\frac{2}{L q} \sin (\mu / 2) \sum_{k=1}^{N_{Q}}(-1)^{k} x_{k} \sin q\left(s-s_{k}\right) H\left(s-s_{k}\right)
$$

Fig. 2 shows this solution for a lattice with a phase advance of $105.5^{\circ}$ and a distance $L=4.56 \mathrm{~m}$ between the quadrupoles as it is the case for second sector of the CLIC main linac. 


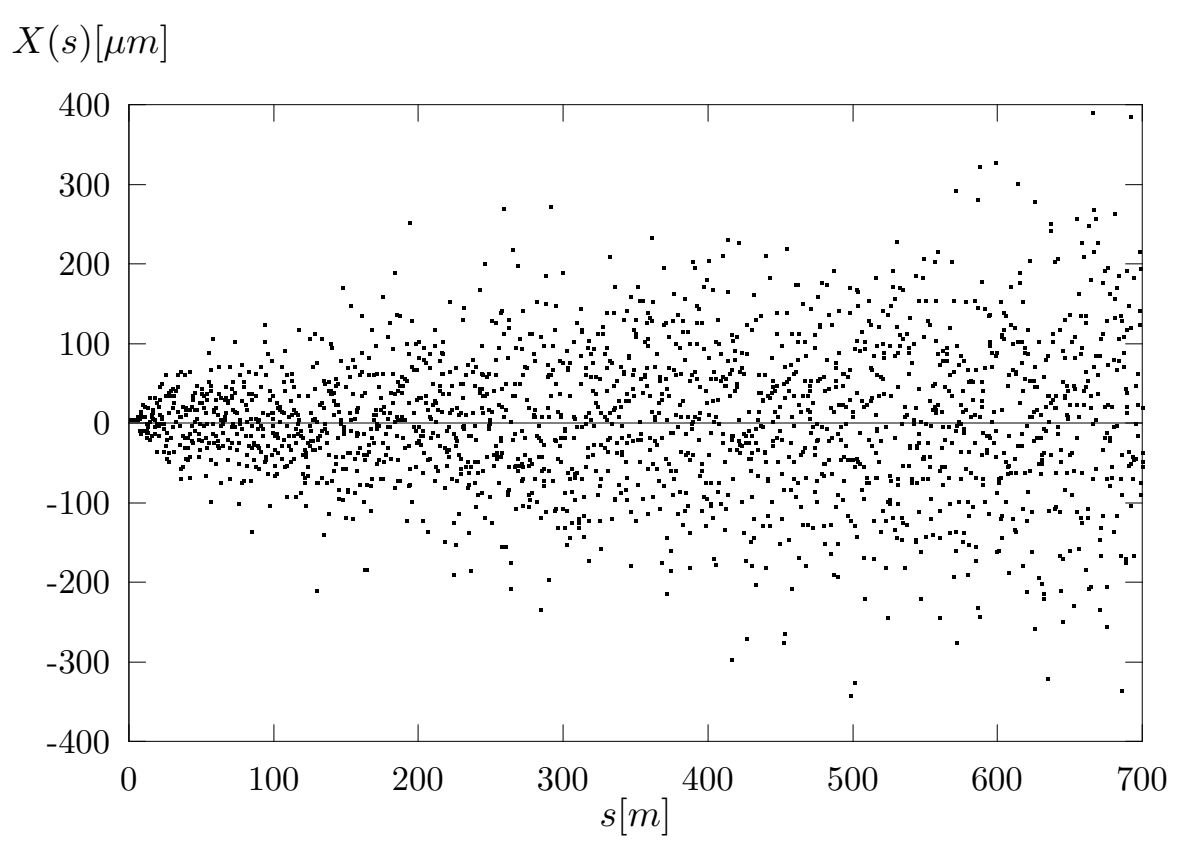

Fig. 2 Solution $X(s)$ under the presence of quadrupole misalignments

In this example we used a uniform random distribution $x_{k}$ with an RMS value of $10 \mu \mathrm{m}$. However, as can be seen, the solution is unbounded and over a distance of only $700 \mathrm{~m}$ the trajectory deviates from the ideal orbit by $0.4 \mathrm{~mm}$ which is equal to 40 times the RMS value of the misalignments. The reason for this behaviour is that the $x_{k}$ are random, meaning that the Fourier spectrum of $x_{k}$ is continuous. Hence every frequency is contained in the oscillatory behaviour of the $x_{k}$ and so the frequency $q$ coming from the right hand side of (41) drives the solution in a resonant way.

\subsection{The equation for the incoherent oscillation $y(s, z)$}

The equation (42) can be written in the form:

$$
\frac{\partial^{2} y}{\partial s^{2}}+\bar{q}^{2}(z) y=A(z) X(s)+K(s) x_{Q}(s) \Delta k(z)++\frac{C W_{0}}{\gamma_{0} l_{B}} \int_{0}^{z} \rho\left(z^{*}\right)\left(z-z^{*}\right) y\left(s, z^{*}\right) d z^{*}
$$

with

$$
A(z)=\left[\frac{C W_{T 0}}{l_{B} \gamma_{0}} \int_{0}^{z} \rho\left(z^{*}\right)\left(z-z^{*}\right) d z^{*}-\left(q^{2}-\bar{q}^{2}(z)\right)\right]
$$

Using the Chebyshev approximation for the gaussian charge distribution $\rho(z)(10)$ the coefficient function $A(z)$ becomes after integrating the fifth order polynomial:

$$
A(z)=\frac{C W_{T 0}}{\gamma_{0}}\left(\frac{16}{23} \zeta^{6}-\frac{48}{23} \zeta^{5}+\frac{79}{46} \zeta^{4}+\frac{1}{23} \zeta^{3}+\frac{3}{23} \zeta^{2}\right)-\left(q^{2}-\bar{q}^{2}(z)\right) \quad ; \quad \zeta=\frac{z}{l_{B}}
$$

Eq. (69) is a linear, second order and inhomogeneous integro-differential equation in two independent variables $s$ and $z$ and with nonconstant coefficients. There is, unfortunatly no closed form solution process known. However, this equation can be treated effectivly by using a perturbation method. We make use of the fact that

- The product of the transverse wake $W_{0}$ and the trajectory deviation $y$ is of second order and 
- The deviation from the nominal focusing $\Delta k(z)$ ocurring in the weak focusing tune $\bar{q}(z ; \Delta k(z))$ is a small perturbation since it is designed precisely for canceling the effect of the (perturbative) wakefield.

We therefore rewrite the above differential equation for $y(s, z)$ as a perturbation problem using the two perturbation parameters $\epsilon$ and $E$ in front of the perturbating terms:

$$
\frac{\partial^{2} y}{\partial s^{2}}+\bar{q}^{2}(z ; E \Delta k(z)) y=A(z) X(s)+K(s) x_{Q}(s) \Delta k(z)+\epsilon \frac{C W_{0}}{\gamma_{0} l_{B}} \int_{0}^{z} \rho\left(z^{*}\right)\left(z-z^{*}\right) y\left(s, z^{*}\right) d z^{*}
$$

We use two formal parameters $E$ and $\epsilon$ but we perform an expansion only with respect to one of the two parameters, namely to $\epsilon$. This method, denoted by partial perturbation expansion, has been introduced in Ref. [1] and which is also described in Appendix $\mathrm{C}$ of this paper. It prevents the occurence of artificial secular terms during the perturbation process because it preserves the detuning due to the perturbation caused by $\Delta k(z)$. In this way a formal perturbation series in $\epsilon$ is

$$
y(s, z)=y^{(0)}(s, z ; E)+\epsilon y^{(1)}(s, z ; E)+\epsilon^{2} y^{(2)}(s, z ; E)+\cdots
$$

To lowest order we then obtain:

$$
\frac{\partial^{2} y^{(0)}}{\partial s^{2}}+\bar{q}^{2}(z) y^{(0)}=X(s) A(z)+K(s) x_{Q}(s) \Delta k(z)
$$

which is a simple, linear, driven harmonic oscillator w.r.t. the variable $s$. Its solution can be written in the following way (superposition of the homogeneous and a particular solution).

$$
y^{(0)}(s, z)=y_{0} \cos \bar{q} s+\frac{y_{0}^{\prime}}{q} \sin \bar{q} s+A(z) G^{\bar{q}}(X)+\Delta k(z) X^{\bar{q}}(s)
$$

where $y_{0}$ and $y_{0}^{\prime}$ are the initial displacement and slope w.r.t $s$ at the entrance of the linac sector. The last term on the right hand side of Eq. (75) comes from a comparison of Eq. (74) to Eq. (41) and it follows that

$$
X^{\bar{q}}(s)=\frac{2}{L \bar{q}} \sin (\mu / 2) \sum_{k=1}^{N_{Q}}(-1)^{k} x_{k} \sin \bar{q}\left(s-s_{k}\right) H\left(s-s_{k}\right)
$$

The operator $G$ applied to a function $g(s)$ is defined in the usual way for linear driven oscillators:

$$
G^{\bar{q}}(g)=\frac{\sin \bar{q} s}{\bar{q}} \int_{0}^{s} \cos \bar{q} s^{*} g\left(s^{*}\right) d s^{*}-\frac{\cos \bar{q} s}{\bar{q}} \int_{0}^{s} \sin \bar{q} s^{*} g\left(s^{*}\right) d s^{*}
$$

Applying this operator to $X(s)$ and inverting summation and integration yields

$$
\begin{aligned}
& G^{\bar{q}}(X)=\sum_{k=1}^{N_{Q}} \frac{2(-1)^{k} x_{k} \sin \bar{q} s}{L q \bar{q}} \sin (\mu / 2) \int_{0}^{s} \cos \bar{q} s \sin q\left(s^{*}-s_{k}\right) H\left(s^{*}-s_{k}\right) d s^{*}- \\
& \sum_{k=1}^{N_{Q}} \frac{2(-1)^{k} x_{k} \cos \bar{q} s}{L q \bar{q}} \sin (\mu / 2) \int_{0}^{s} \sin \bar{q} s \sin q\left(s^{*}-s_{k}\right) H\left(s^{*}-s_{k}\right) d s^{*}
\end{aligned}
$$

We now make use of the following identity which is demonstrated in Appendix B:

$$
\int_{0}^{s} f\left(s^{*}\right) H\left(s^{*}-s_{k}\right) d s^{*}=H\left(s-s_{k}\right) \int_{s_{k}}^{s} f\left(s^{*}\right) d s^{*}
$$


In this way we obtain:

$$
\begin{aligned}
& G^{\bar{q}}(X)=\sum_{k=1}^{N_{Q}} \frac{2(-1)^{k} x_{k} \sin \bar{q} s}{L q \bar{q}} \sin (\mu / 2) H\left(s-s_{k}\right) I_{1}\left(s, s_{k}\right)- \\
& \sum_{k=1}^{N_{Q}} \frac{2(-1)^{k} x_{k} \cos \bar{q} s}{L q \bar{q}} \sin (\mu / 2) H\left(s-s_{k}\right) I_{2}\left(s, s_{k}\right)
\end{aligned}
$$

with

$$
\begin{aligned}
& I_{1}\left(s, s_{k}\right)=\int_{s_{k}}^{s} \cos \bar{q} s^{*} \sin q\left(s-s_{k}\right) d s^{*} \\
& I_{2}\left(s, s_{k}\right)=\int_{s_{k}}^{s} \sin \bar{q} s^{*} \sin q\left(s-s_{k}\right) d s^{*}
\end{aligned}
$$

Evidently these integrals can be evaluated in an elementary way and thus the total solution for $y^{(0)}(s, z)$ can be written in a closed form:

$$
\begin{aligned}
& y^{(0)}(s, z)=y_{0} \cos \bar{q}(z) s+\frac{y_{0}^{\prime}}{\bar{q}(z)} \sin \bar{q}(z) s \\
& A(z) \sum_{k=1}^{N_{Q}} \frac{2}{L q \bar{q}(z)} \sin (\mu / 2)(-1)^{k} x_{k} H\left(s-s_{k}\right)\left[\sin \bar{q}(z) s I_{1}\left(s, s_{k}\right)-\cos \bar{q}(z) s I_{2}\left(s, s_{k}\right)\right] \\
& +\Delta k(z) \frac{2}{L q \overline{(} z)} \sin (\mu / 2) \sum_{k=1}^{N_{Q}}(-1)^{k} x_{k} \sin \bar{q}(z)\left(s-s_{k}\right) H\left(s-s_{k}\right)
\end{aligned}
$$

The evaluation of $I_{1,2}$ gives

$$
\begin{aligned}
& I_{1}\left(s, s_{k}\right)=\frac{q\left[2 \cos \bar{q}(z) s_{k}-\cos \left((q-\bar{q}(z)) s-q s_{k}\right)-\cos \left((q+\bar{q}(z)) s-q s_{k}\right)\right]}{2\left(q^{2}-\bar{q}^{2}(z)\right)}+ \\
& +\frac{\bar{q}(z)\left[-\cos \left((q-\bar{q}(z)) s-q s_{k}\right)+\cos \left((q+\bar{q}(z)) s-q s_{k}\right)\right]}{2\left(q^{2}-\bar{q}^{2}(z)\right)} \\
& I_{2}\left(s, s_{k}\right)=\frac{q\left[2 \sin \bar{q}(z) s_{k}+\sin \left((q-\bar{q}(z)) s-q s_{k}\right)-\sin \left((q+\bar{q}(z)) s-q s_{k}\right)\right]}{2\left(q^{2}-\bar{q}^{2}(z)\right)}+ \\
& +\frac{\bar{q}(z)\left[\sin \left((q-\bar{q}(z)) s-q s_{k}\right)+\sin \left((q+\bar{q}(z)) s-q s_{k}\right)\right]}{2\left(q^{2}-\bar{q}^{2}(z)\right)}
\end{aligned}
$$

These expressions exhibit clearly the near resonant dynamics of the single bunch problem with wakefields. Depending on the position $z$ inside the bunch the denominators $q^{2}-\bar{q}^{2}(z)$ deviate from zero in a certain way (due to a finite correcting force $\Delta k(z)$ originating from the correlated energy spread and/or RF quadrupoles) but always remain small. For $z=0$ however, $q=\bar{q}$ and the situation becomes exactly resonant. This, on the contrary does not lead to an unbounded trajectory since the coefficient $A(z)$ in (83) becomes zero in this case as can be seen from (71).

As will be shown in Appendix B there exists a method to represent the Heaviside function contained in the above expressions as a trigonometric series in $s-s_{k}$. In this representation it becomes possible to express $y$ in terms of sums of products of functions of $s$ and $s_{k}$ only. However, since the following relations hold,

$$
\begin{aligned}
& \sin a\left(s-s_{k}\right)=\sin a s \cos a s_{k}-\cos a s \sin a s_{k} \\
& \cos a\left(s-s_{k}\right)=\cos a s \cos a s_{k}+\sin a s \sin a s_{k}
\end{aligned}
$$


all terms that only depend on $s$ can then be written in front of the summation symbols over the quadrupole index $k$. These remaining sums can then be computed at once for a given set of machine parameters before evaluating $y(s, z)$ for a specific value of $s$, which simplifies and speeds up the treatment.

The emittance growth of the bunch at any position $s$ along the linac can be computed using the relation:

$$
\Delta\left(\gamma \epsilon_{y}\right)=\gamma_{0} \int_{0}^{l_{B}} \rho(z)\left[q y^{2}(s, z)+\frac{1}{q}\left(\frac{\partial y}{\partial s}(s, z)\right)^{2}\right] d z
$$

This relation gives the contribution to the normalised emittance of the bunch center-of-gravity off-sets due to wakefields and quadrupole misalignments. Computing the total emittance requires to add the normalised emittance given at injection. It has to be noted that the contributions due to the dispersion are included in the above treatment (via $\delta(s, z)$ in the orbit calculations), but not those of the accelerating structure misalignments, filamentation due to chromaticity and particle scattering, which come in addition.

\section{Trajectory correction and time-dependent misalignment}

The final expression for the incoherent off-sets $y_{0}(s, z)$ within the bunch contains a sum over all the quadrupoles of a linac sector and each term of the sum is proportional to the quadrupole displacement $x_{k}$. Hence, the result can directly be used to predict the effects of different kinds of quadrupole displacements, either correlated or uncorrelated, provided that there exists a function that describes the $x_{k}$ or a recurrence relation which allows to deduce them through an iterative computation. In this section, we shall explain how it is possible to include in this analytical model the displacements due to random misalignments, re-alignments based on beam position measurements and drifts due to ground motion.

\subsection{Quadrupole random misalignments}

It is generally admitted that after the pre-alignment of the linac components, the quadrupole transverse positions are randomly distributed following a gaussian distribution of given r.m.s. value (typically $10 \mu \mathrm{m}$ ). Since it is not convenient for an analytical approach to use a numerical generator of random numbers, it becomes necessary to find out a function of the index $k$ capable to generate a sequence of random numbers $x_{k}^{r}$. This function must also depend on a parameter in such a way that a small variation of it orginates into a totally different sequence. Assuming such a function exists, the actual randomness of the numbers obtained has to be checked from the auto-correlation function (Appendix $\mathrm{C}$ ) which must contain a narrow peak at the origin and a residual amplitude on both sides at least 10 times smaller than the peak amplitude.

The theory of chaos [18] is deeling with nonlinear maps that may generate chaotic behaviour of the variables and therefore provide a possible receipe to construct a function generating random numbers. The idea mentioned here is issued from the properties of the logistic map [18], that is a one-dimensional, non-linear map, with a mapping parameter $\mu$, and is defined as follows

$$
x_{k+1}=\mu x_{k}\left(1-x_{k}\right)
$$

Considering the particular value $\mu=4$ of the mapping parameter, the map (89) has a closed algebraic solution which contains a sinusoidal function of $2^{k}$ and of an initial value $\theta_{0}$

$$
x_{k}=\left(\sin \theta_{0} 2^{k}\right)^{2}
$$


The solution (90) has a chaotic behaviour which can be established from the Lyapounov coefficient theory (Appendix D). Developing this power of 2 into a polynomial series indicates that the sinus function of a monome of degree 2 or more might also provide a series of $x_{k}$-values with chaotic behaviour. Hence, a possible function likely to generate a series of random numbers (positive as well as negative) might look like:

$$
x_{k}^{r} \approx \sin \left(a k^{2}\right)
$$

The next step consists of checking the randomness of the points $x_{k}^{r}$ by looking at the autocorrelation characteristic of (91), which is discussed in the Appendix C. It is also shown there that the sequences obtained with slightly different values of $a$ are totally unlike. This function provides a distribution which is approximately rectangular with rising edges. However a combination of different sinus functions of different polynoms allows to modify the distribution in order to get for instance an approximation of the gaussian which is generally used.

Once a sequence of random quadrupole misalignmants is obtained by using (91), the explicit form (68) of the solution for the betatron motion $X(s)$ provides the average bunch position at any position $s$ along the linac. In particular, it gives the off-set average-amplitude at every beam position monitor (or pick-up), $x_{P U}^{m}$, termed here the measured trajectory off-set ("measured" with respect to the $s$-axis of the coordinate system of reference).

\subsection{Quadrupole displacements after trajectory correction}

\subsubsection{Balistic correction}

In the balistic [19] correction, the quadrupoles of a sector bin are switched off and the beam shooted through. The beam, centred in the pick-up that stands at the beginning of the bin by a preceding adjustment, is also centred in the pick-up at the bin end by moving the first quadrupole of the bin. The beam defines itself a straight line on which all the intermediate position monitors can be aligned, using direct beam measurements. This means we can compute all pick-up positions from the following relation (neglecting the monitor resolution):

$$
x_{P U j}^{c}=x_{P U 1}^{r}+\frac{j-1}{n}\left(x_{P U n}^{r}-x_{P U 1}^{r}\right)
$$

where $P U 1$ and $P U n$ are the first and the last pick-up of the bin, the superscript $\mathrm{r}$ denotes a random value (initial position) and c a correlated value after beam based re-alignment. The corresponding additional displacement of the first quadrupole, effectively sending the beam on the straight line defined by (92), results from

$$
\Delta x_{Q 1, j}^{c}=\frac{f}{n}\left(\frac{\left(x_{P U n, j}^{r}-x_{P U 1, j}^{r}\right)}{L_{b i n, j}}-\frac{\left(x_{P U n, j-1}^{r}-x_{P U 1, j-1}^{r}\right)}{L_{b i n, j-1}}\right)
$$

where the additional index $\mathrm{j}$ or $\mathrm{j}-1$ refers to the bin considered, $f$ is the focal distance of the quadrupoles, $L_{b i n, j}$ and $L_{b i n, j-1}$ are the lengths of the bins $j$ and $j-1$ respectively. The expression (93) directly provides the movement resulting from a balistic correction that we have to include into the $x_{k}$ values of the quadrupole displacements. Note that every $n-t h$ quadrupole 
is moved when the balistic correction is carried on, while the others will only be re-positioned when a correction of the type described in the next section is applied.

\subsubsection{One-by-one trajectory correction}

Once the balistic correction has been done, all the quadrupoles are switched on again to their nominal values. The beam trajectory does not follow the ideal straight line (92) anymore, but can be brought toward this line with a one-by-one correction. This commonly used method centers the beam in each pick-up successively, by moving the quadrupole that immediately precedes it, according to

$$
\Delta x_{k}=-\frac{f_{k}}{L} \Delta x_{P U k+1}
$$

where $f_{k}$ represents the focal distance of the quadrupole $k, L$ the distance between two consecutive quadrupoles and $\Delta x_{P U k+1}$ is the change of trajectory to achieve at the pick-up $k+1$. This change is given by the measured off-set $x_{P U}^{m}$ (all quadrupoles ON), corrected for the displacement $x_{P U}^{c}$ bringing the pick-up on the balistic line (92). Taking into account in addition the effects of re-positioning all the quadrupoles which precede the quadrupole $k$ considered, the change of trajectory to be achieved becomes

$$
\Delta x_{P U k+1}=x_{P U k+1}^{m}-x_{P U k+1}^{c}+\sum_{j=1}^{k} \Delta x_{P U k+1}\left(Q_{j}\right)
$$

The last term represents the beam-deviation amplitude at the pick-up $k+1$ due to the displacement $\Delta x_{j}$ of any preceding quadrupole $Q_{j}$ and can be written in a closed form

$$
\Delta x_{P U k+1}\left(Q_{j}\right)=\frac{\Delta x_{j}}{f_{j}} \sin \left[q\left(s_{k+1}-s_{j}\right)\right]
$$

provided the small effects of the wakefields on the average trajectory is neglected, assumption which is certainly acceptable. Putting together the last three relations (94),(95) and (96), we can write a complete expression for the quadrupole movements associated with a one-by-one correction,

$$
\Delta x_{k}=-\frac{f_{k}}{L}\left(x_{P U k+1}^{m}-x_{P U k+1}^{c}+\sum_{j=1}^{k-1} \frac{\Delta x_{j}}{f_{j}} \sin \left[q\left(s_{k+1}-s_{j}\right)\right]\right)
$$

where $f_{j}$ takes the values $f(-1)^{j}$ in a constant FODO lattice. The relation (97) is nothing else but a recurrence relation which takes the form

$$
\begin{aligned}
& \Delta x_{1}=-\frac{f}{L}\left(x_{P U 2}^{m}-x_{P U 2}^{c}\right) \\
& \Delta x_{2}=\frac{f}{L}\left(x_{P U 3}^{m}-x_{P U 3}^{c}+\frac{\Delta x_{1}}{f} \sin \left[q\left(s_{3}-s_{1}\right)\right]\right) \\
& \Delta x_{3}=-\frac{f}{L}\left(x_{P U 4}^{m}-x_{P U 4}^{c}+\frac{\Delta x_{1}}{f} \sin \left[q\left(s_{4}-s_{1}\right)\right]-\frac{\Delta x_{2}}{f} \sin \left[q\left(s_{4}-s_{1}\right)\right]\right) \\
& \ldots
\end{aligned}
$$


We recall the reader that the "correlated" quantities $x_{P U}^{c}$ are defined in eq. (92) and contain a certain number of random numbers (used to define the successive balistic lines) which can be expressed via a function of the form (91). Hence, all the necessary quantities $x_{P U}, x_{k}^{r}$ and $\Delta x_{k}$ associated with both the balistic and the one-by-one corrections can be written in an algebraic form that can be used in the formula (83) for the uncoherent off-set $y^{(0)}(s, z)$.

It would not be difficult, though more cumbersome, to add small random quantities to all the off-sets measured at the beam position monitors as well as to the calculated quadrupole displacements resulting from the trajectory correction. Using again functions of the type (91) for generating these values, the effect of the pick-up and micromover resolutions could also be modelled analytically.

\subsubsection{Quadrupole drifts due to ground motion}

The commonly used model for drifts due to ground motion is the so-called ATL law which defines the transverse displacements $d x$ of different elements of the linac separated by a distance $L$ and a time interval T. More precisely, the resulting random walk of two distinct components of a linac is given in this model by the double-average over the time $t$ and the distance $s$ of the second-order infinitesimal difference defined as follows:

$$
<<([d x(s+L, t+T)-d x(s+L, t)]-[d x(s, t+T)-d x(s, t)])^{2}>>=A \cdot T \cdot L
$$

The transverse displacement $d x$ is taken at tow different instants $t$ and $t+T$, and two different positions $s$ and $s+L$. The constant A takes values between $10^{-7}$ and $10^{-5} \mu m^{2} s^{-1} m^{-1}$, depending on the nature and stability of the ground.

Applying this ATL law to two consecutive quadrupoles of a linac sector, separated by a distance L, and using finite differences $\Delta x$, the relation (99) becomes after taking the average and the square root

$$
\Delta x_{k}(T)-\Delta x_{k}(0)=\Delta x_{k-1}(T)-\Delta x_{k-1}(0)+g_{k} \sqrt{A \cdot T \cdot L}
$$

Because of the random nature of the process, the quantity $g_{k}$ is a gaussian variable of zero average and standard-deviation equal to one.

This relation is again equivalent to a recurrence relation which looks as follows, if we assume that the first element does not move:

$$
\begin{aligned}
& \Delta x_{1}(T)=\Delta x_{1}(0) \\
& \Delta x_{2}(T)=\Delta x_{2}(0)+g_{2} \sqrt{A \cdot T \cdot L} \\
& \Delta x_{3}(T)=\Delta x_{3}(0)+g_{2} \sqrt{A \cdot T \cdot L)}+g_{3} \sqrt{A \cdot T \cdot L}=\left(g_{2}+g_{3}\right) \sqrt{A \cdot T \cdot L} \\
& \ldots
\end{aligned}
$$

This recurrence (101) provides the contributions to be added to the quadrupole displacements $x_{k}$ in order to simulate the slow drift due to the ground motion. Here again, the random values $g_{k}$ could be deduced from an algebraic function of the type (91). Note that, in the particular case 
of random walk due to ground motion, the recurrence simply reduces to a successive addition of random numbers selected in a distribution of average equal to zero and standad-deviation equal to one.

\section{Comparison of the analytical results to MUSTAFA}

In order to demonstrate that the analytical results obtained in this paper are describing correctly the motion of a single bunch in a linac with wakefields, we now compare them to tracking results obtained with the MUSTAFA code [2]. For this purpose we first consider a case with a highly charged bunch $\left(q=6 \cdot 10^{9}\right.$ particles per bunch), a bunchlength of $0.2 \mathrm{~mm}$ and a distance between quadrupoles of $2.28 \mathrm{~m}$ over a fictitious linac sector of $1000 \mathrm{~m}$. Compared to the present CLIC main linac design these parameters are quite strong meaning that the wakefield effects act strongly on the bunch. The RMS value for the randomly distributed quadrupole misalignments has been set to $10 \mu \mathrm{m}$. Figs. 3 to 5 show $y(s, z)$ for the three distances of $s=100 \mathrm{~m}, s=500 \mathrm{~m}$ as well as $s=1000 \mathrm{~m}$ and for a setting of the RF quadrupole strength of $\alpha_{0}=0.2$. Due to the force depending on the position $z$ inside the bunch, different parts of it oscillate with different frequencies and the motion is progessively decohered. Therefore a typical oscillation inside the bunch w.r.t $z$ can be observed whose frequency increases as we proceed downstream the linac. In adition the oscillation amplitude increases with $s$ which is due to the betatron motion which builds up under the effect of the randomly misaligned quadrupoles.

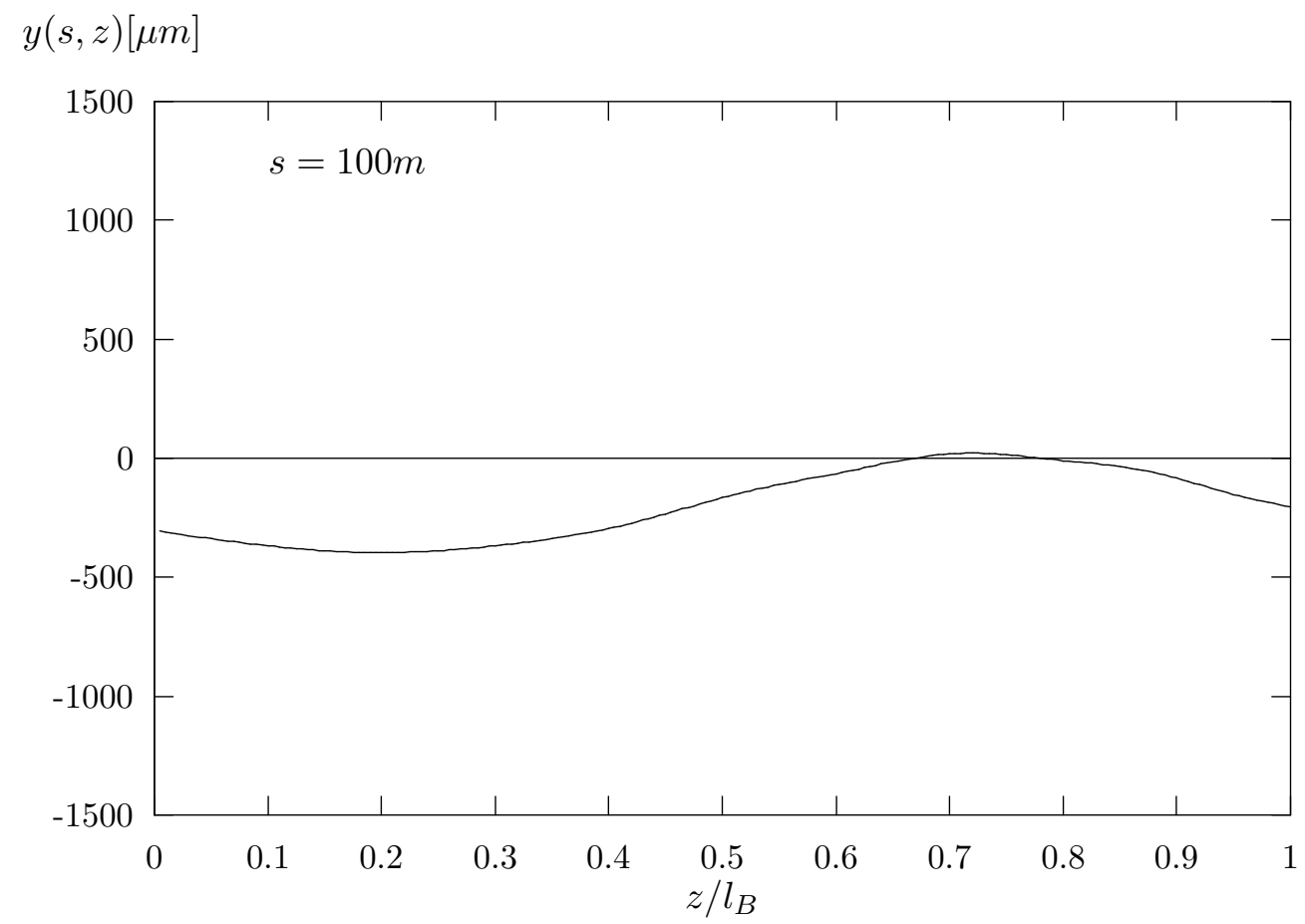

Fig. 3 Displacement of the bunch as function of $z$ at $s=100 \mathrm{~m}$ 


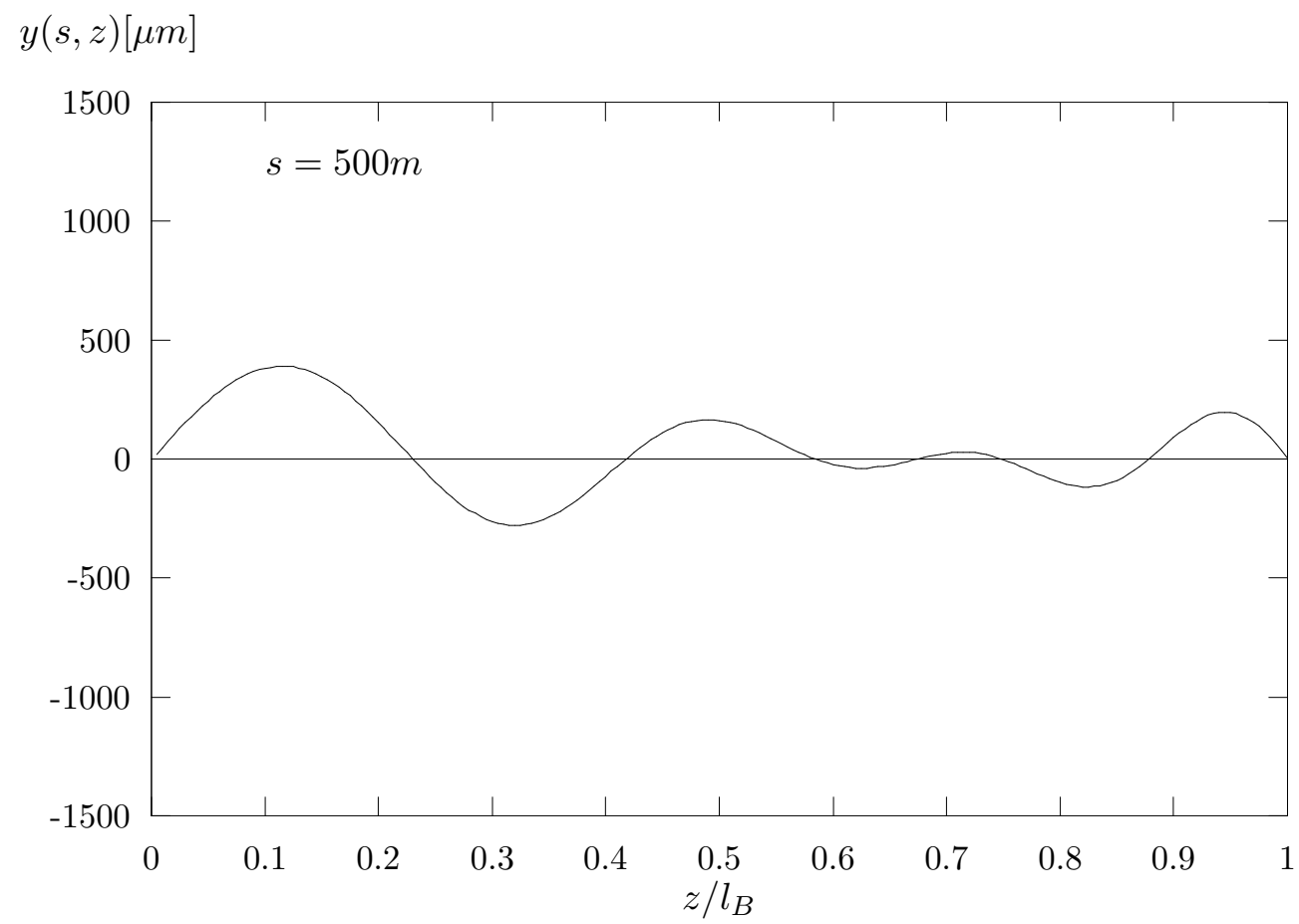

Fig. 4 Displacement of the bunch as function of $z$ at $s=500 \mathrm{~m}$

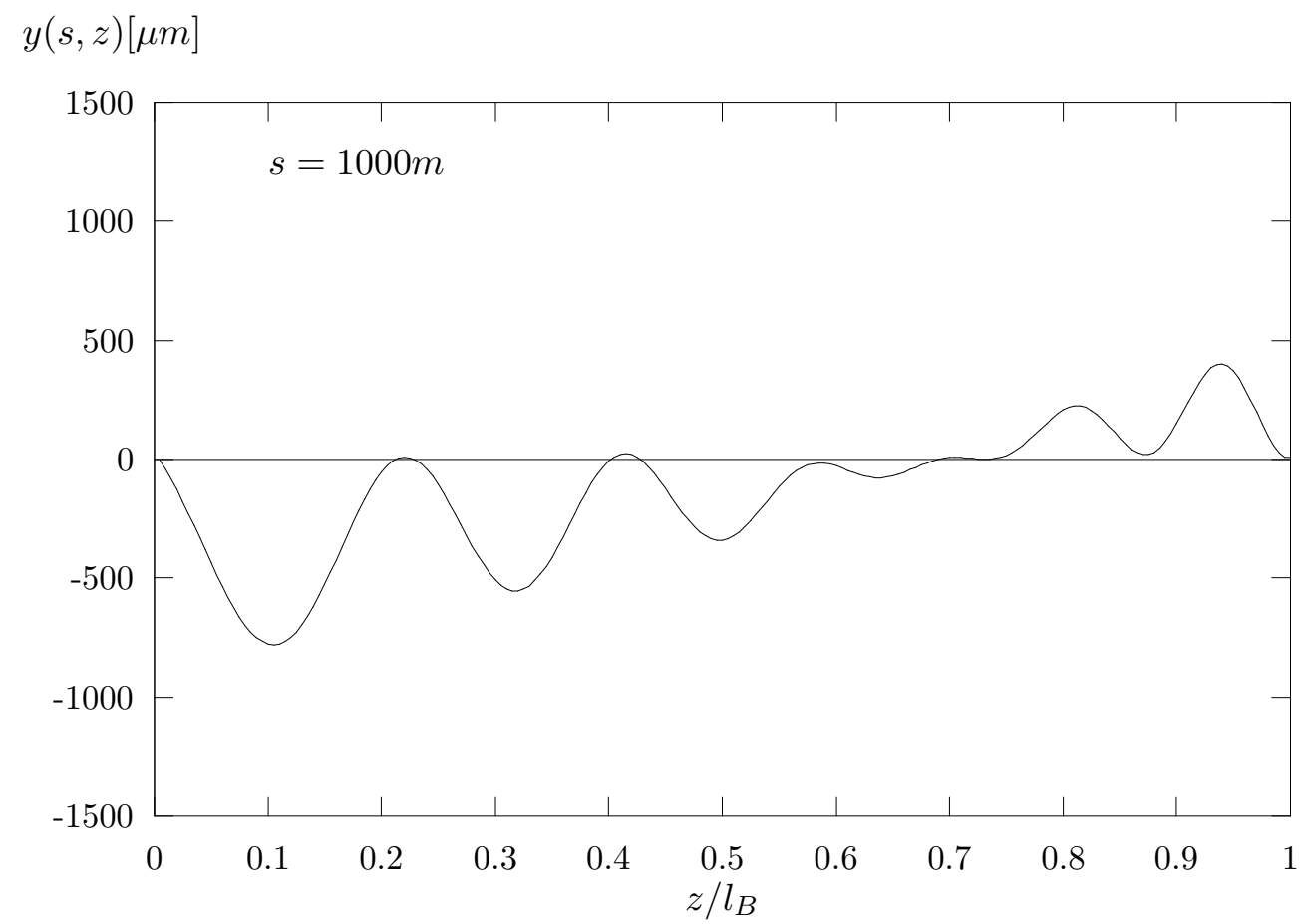

Fig. 5 Displacement of the bunch as function of $z$ at $s=1000 \mathrm{~m}$ 
We finally present the results of a fully numerical integration by MUSTAFA of the same particular case, after $1000 \mathrm{~m}$ and using the same machine parameters (Fig. 6).

It can be seen that the numerical solution agrees in frequency and amplitude with the analytical one. Small remaining differences are due to the fact that contrary to tracking we use a weak focusing model for the betatron motion and in addition derive the random misalignments from an analytical formula given by (see Appendix D)

$$
x_{k}=2<x>\sin \left(a k^{2}\right)
$$

instead of the recursive method used by MUSTAFA for the same purpose.

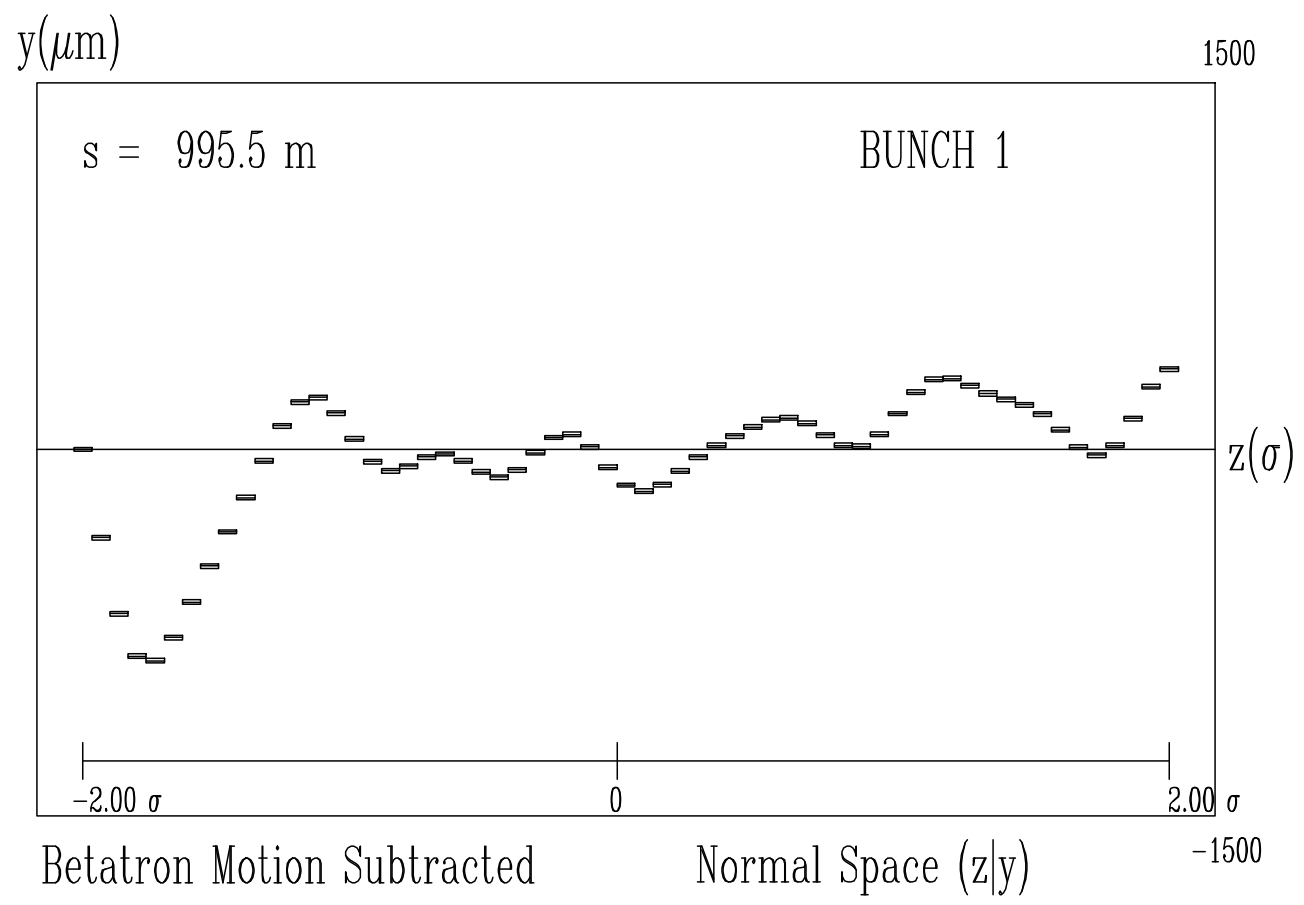

Fig. 6 Numerical results at $s=1000 \mathrm{~m}$ obtained by MUSTAFA 


\section{Conclusions}

In this paper we succeeded to present a fully analytical treatment of the motion of a bunch of charged particles inside a linac with longitudinal and transverse wakefields as well as random quadrupole misalignments. This goal has been achieved by directly solving the longitudinal and transverse equations of motion for any particle inside the bunch. While the longitudinal equation provided the form of the wakefield is given - can be solved in a straightforward way, the transverse equation is a linear, second order, partial integro-differential equation in two variables and with nonconstant coefficients and its solution can only be found by application of a perturbation technique. A completly new type of perturbation technique denoted by "Partial Perturbation Expansion" has been developed which enables us to find perturbation equations that are free of secular terms and thus lead at any order to uniformally valid approximations for the solution. In addition we had to introduce a formalism for dealing with the equation of betatron motion in the presence of quadrupole misalignments. While the misalignments themselves can successfully be modeled by application of chaos-theoretical concepts (leading to trigonometric functions of polynomial arguments), the oscillator equation driven by random kicks can be tackled by applying the superposition principle and the Heaviside function formalism. In this way and by assuming a weak focusing model for the betatron motion the perturbation equations become linear and identical to those of driven harmonic oscillators and can be solved by the standard Green's techniques. The so obtained analytical expressions have been physically interpreted and describe the effect of single bunch break-up under the presence of wakefields. This manifests itself by more and more center-of-gravity wiggles along the bunch as it proceeds down the linac. Also the effect of a correction due to BNS damping either by RF quadrupoles or by a variation of the RF phase can well be described and understood using this analytical formalism. Furthermore we derived a sequence of algorithms dealing with correction schemes for the quadrupole misalignments in a linac and including the ATL law related to ground motion.

The results obtained in this work can be used to obtain a deeper understanding of the underlying physics driving the effect of beam breakup in linacs. In addition the dependence of this effect on the basic machine parameters like charge of the bunch, frequency of the RF system, RMS value of the element misalignments and so on can be clearly extracted.

The analytical expression for the transverse displacement $y(s, z)$ of the bunch center of gravities can also provide a closed expression for the emittance increase due to wakefields as function of the position $s$ inside the linac as well as of the machine parameters. In addition we plan to use the analytic expression for $y$ as a complement to the numerical integration by the tracking facility of the MUSTAFA environment. This will enable us to obtain preliminary results for the emittance increase in a much faster way (about a factor 20) compared to numerical integration of the equation of motion. 


\section{References}

[1] G. Guignard and J. Hagel, Analytical Treatment of Single Bunch Transverse Dynamics in Linacs with Wakefields, CERN-SL-98-015 (AP) and CLIC Note 362 (1998).

[2] G. Guignard and J. Hagel, "MUSTAFA Environment Description and users' Guide with Applications to CLIC (Version 1.0)", CERN SL 98-002 (AP) and CLIC-Note-349 (1998).

[3] W.K.H. Panofsky and M. Bander, Rev. Sc. Instrum.,39, 206 (1968).

[4] V.K. Neil, L.S. Hall and R.K.Cooper, Part. Acc., 9, 213 (1979).

[5] A.W. Chao, B. Richter and C.Y. Yao, "Beam Emittance Growth Caused by Transverse Deflecting Fields in a Linear Accelerator", Nucl. Instrum. Methods, 178, 1 (1980).

[6] K.L.F. Bane, "Wakefield Effects in a Linear Collider", SLAC PUB 4169 (1986).

[7] V. Balakin, A. Novokhatsky and V. Smirnov, "Longitudinal Dynamics in the VLEPP Collider", Proc. of the 12th Int. Conf. on High Energy Accel., Fermilab, Batavia, IL, 119, 1983 (edited in 1984).

[8] H. Henke and W. Schnell, "An Analytical Criterion for the Onset of Transverse Damping due to Wakefields in a Linear Accelerator", report CERN-LEP-RF/86-18 and CLIC Note 22, CERN (1986).

[9] R.L. Gluckstern, F. Neri, and J.B.J. van Zeijts, "Suppression of Single Bunch Beam Breakup by BNS Damping", Proc. of the Linear Accel. Conf., Albuquerque, NM, 309 (1990); and R.L. Gluckstern, "Compensation of Single Bunch Transverse Beam Brea-kup in a Chain of Long periodic Linac Cavities", CLIC Note 128, CERN (1990).

[10] V. Balakin, Proc. of the 1988 International Workshop on Linear Colliders, SLAC-report-355, 55 (1988).

[11] T.O. Raubenheimer and K. Kubo, "A technique of measuring and Correcting Emittance Dilutions due to Accelerator Structure Misalignments", SLAC PUB 95-6523 (1995).

[12] G.V. Stupakov, "BNS Damping of Beam Break-up Instability", SLAC-AP-108 (1997).

[13] Y. Chin, report CERN-SPS/86-05 (1986), and K. Yokoya, report DESY 86-084 (1986).

[14] A.W. Chao, unpublished, and in "Physics of Collective Beam Instabilities in High Energy Acelerators", ed. by John Wiley \& Sons, Inc., NY (1993).

[15] D. Chernin an A. Mondelli, "Effect of Energy Spread on the Single-Bunch Dipole Beam Break-up Instability in a High-Energy RF Linac", Part. Acc., 24, 177, (1989).

[16] Bruce W. Char et al., "MAPLE V, A tutorial Introduction", Springer Verlag (1992).

[17] T.O. Raubenheimer, "Summary of formula for emittance dilution in high energy linacs", CLIC-Note-347 (1997)

[18] S. Neil Rasband, "Chaotic Dynamics of Nonlinear Systems", department of Physics and Astronomy, Wiley-Interscience-Pubilication.

[19] D. Schulte, "The CLIC main linac lattice at 1 TeV", CLIC-Note-356 (1998).

[20] J. Hagel, B. Zotter, "A new approach to potential well bunch deformation", CERN SL/91-07 (AP) (1991). 


\section{A Solution of the betatron equation with dipole kicks}

The betatron equation that we have to solve and the Green function associated with a single unity dipole-kick are recalled hereafter:

$$
\begin{gathered}
\frac{d^{2} X(s)}{d s^{2}}+q^{2} X(s)=\theta(s) \\
\frac{\partial^{2} G\left(s, s^{*}\right)}{\partial s^{2}}+q^{2} G\left(s, s^{*}\right)=\delta\left(s-s^{*}\right)
\end{gathered}
$$

Since the number of kicks is unlimited and they can be continously distributed along $s$, the function $\theta(s)$ is continuous in general. We have seen in the Section 3.1 that the solution of the equation for the Green function (response to a unity-kick at any arbitrary poition $s^{*}$ ) can be written,

$$
G\left(s, s^{*}\right)=\frac{1}{q} \sin \left[q\left(s-s^{*}\right)\right] H\left(s-s^{*}\right)
$$

Given the symmetry properties of the function $G(s, s *)$, we can write after inverting the coordinates $s$ and $s^{*}$ :

$$
\begin{aligned}
& G\left(s^{*}, s\right)=\frac{1}{q} \sin \left[q\left(s^{*}-s\right)\right] H\left(s^{*}-s\right)= \\
& =-G\left(-s,-s^{*}\right)=G\left(s, s^{*}\right)
\end{aligned}
$$

Starting from the two relations recalled at the beginning of this appendix, let us multiply (103) by the Green function $G\left(s, s^{*}\right)$, rewrite (104) for $G\left(s^{*}, s\right)$ instead of $G\left(s, s^{*}\right)$ and multiply the equation obtained by $X\left(s^{*}\right)$. This gives

$$
\begin{gathered}
G\left(s, s^{*}\right) \frac{d^{2} X\left(s^{*}\right)}{d s^{* 2}}+q^{2} G\left(s, s^{*}\right) X\left(s^{*}\right)=G\left(s, s^{*}\right) \theta\left(s^{*}\right) \\
X\left(s^{*}\right) \frac{\partial^{2} G\left(s^{*}, s\right)}{\partial s^{* 2}}+X\left(s^{*}\right) q^{2} G\left(s^{*}, s\right)=X\left(s^{*}\right) \delta\left(s-s^{*}\right)
\end{gathered}
$$

Subtracting term by term the last two equations, it is straightforward to write the following,

$$
\begin{aligned}
& X\left(s^{*}\right) \frac{\partial^{2} G\left(s^{*}, s\right)}{\partial s^{* 2}}-G\left(s, s^{*}\right) \frac{d^{2} X\left(s^{*}\right)}{d s^{* 2}}+q^{2}\left[G\left(s^{*}, s\right)-G\left(s, s^{*}\right)\right] X\left(s^{*}\right)= \\
& =X\left(s^{*}\right) \delta\left(s-s^{*}\right)-G\left(s, s^{*}\right) \theta\left(s^{*}\right)
\end{aligned}
$$

After taking into account the symmetry properties (106), the last realation simplifies to

$$
\begin{aligned}
& X\left(s^{*}\right) \frac{\partial^{2} G\left(s^{*}, s\right)}{\partial s^{* 2}}-G\left(s, s^{*}\right) \frac{d^{2} X\left(s^{*}\right)}{d s^{* 2}}= \\
& =X\left(s^{*}\right) \delta\left(s-s^{*}\right)-G\left(s, s^{*}\right) \theta\left(s^{*}\right)
\end{aligned}
$$

The next step consists of integrating the differential equation (110) over the coordinate $s^{*}$, between the limits 0 and $s$. The first two terms can be integrated by parts, which gives 


$$
\begin{aligned}
& \int_{0}^{s} X\left(s^{*}\right) \frac{\partial^{2} G\left(s^{*}, s\right)}{\partial s^{* 2}} d s^{*}=\left[X\left(s^{*}\right) \frac{\partial G\left(s^{*}, s\right)}{\partial s^{*}}\right]_{0}^{s}-\int_{0}^{s} \frac{\partial G\left(s^{*}, s\right)}{\partial s^{*}} \frac{d X\left(s^{*}\right)}{d s^{*}} d s^{*} \\
& \int_{0}^{s} \frac{d^{2} X\left(s^{*}\right)}{d s^{* 2}} G\left(s, s^{*}\right) d s^{*}=\left[G\left(s, s^{*}\right) \frac{d X\left(s^{*}\right)}{d s^{*}}\right]_{0}^{s}-\int_{0}^{s} \frac{\partial G\left(s, s^{*}\right)}{\partial s^{*}} \frac{d X\left(s^{*}\right)}{d s^{*}} d s^{*}
\end{aligned}
$$

Subtracting these two contributions, as in (110), making use of (106) and integrating the right hand side of the same equation (110), we obtain,

$$
\begin{aligned}
& {\left[X\left(s^{*}\right) \frac{\partial G\left(s^{*}, s\right)}{\partial s^{*}}-G\left(s^{*}, s\right) \frac{d X\left(s^{*}\right)}{d s^{*}}\right]_{0}^{s}=} \\
& =\int_{0}^{s}\left[X\left(s^{*}\right) \delta\left(s-s^{*}\right)-G\left(s, s^{*}\right) \theta\left(s^{*}\right)\right] d s^{*}
\end{aligned}
$$

The explicit form (106) of $G\left(s^{*}, s\right)$ clearly indicates that the Green function and its derivative with respect to $s^{*}$ are both periodic with a period $\lambda_{\beta}=\frac{2 \pi}{q}$. Let us therefore perform the integral (112) over a period $\lambda_{\beta}$ or any multiple of the period $n \lambda_{\beta}$, taking benefit from the following identity

$$
\int_{0}^{n \lambda_{\beta}} \frac{\partial}{\partial s^{*}}\left(X \frac{\partial G}{\partial s^{*}}-G \frac{d X}{d s^{*}}\right) d s^{*} \equiv 0
$$

Integrating (112) in the interval $\left[0, n \lambda_{\beta}\right]$ and making use of (113) provides,

$$
0=X(s)-\int_{0}^{n \lambda_{\beta}} G\left(s, s^{*}\right) \theta\left(s^{*}\right) d s^{*}
$$

which eventually leads to a closed expression for the solution $X(s)$, i.e. the betatron motion due to continuously distributed dipole kicks

$$
X(s)=\int_{0}^{n \lambda_{b e}} G\left(s, s^{*}\right) \theta\left(s^{*}\right) d s^{*}=\int_{0}^{n \lambda_{b e}} \frac{\theta\left(s^{*}\right)}{q} \sin \left[q\left(s-s^{*}\right)\right] H\left(s-s^{*}\right) d s^{*}
$$

This derivation establishes the correct form of the solution of the first equation (103), solution which is used in eq. (47) of Section 3.1. This result shows in particular that the solution $X(s)$ varies in amplitude and angle when the integration is done over a finite interval of distributed kicks. Therefore, the condition of constant amplitude, which is necessary for the superposition principle described in Section 3.1 to apply, is only satisfied for an infinitely thin dipole, but not for a long displaced quadrupole. 


\section{B Properties and approximation of the Heaviside function}

The advantage resulting of separating all the terms depending on the index $k$ of the quadrupole displacements and summing them up independently of the form of the complete explicit solution is mentioned in Section 3. It is also said that this means to replace the functions of $\left(s-s_{k}\right)$ by products of functions of $s$ with functions of $s_{k}$.

The basic idea to reach this condition is to approximate Heaviside's function by a series of continuous functions in an intervall larger than the length of a sector $L_{S}$. The shortest possible intervall to be considered corresponds to twice the total length $L_{S}$, so as to proprely cover the cases where the kick applies either at the beginning or the end of the sector. There is no significant improvement of the approximation by extending the interval because we then loose on the sharpness of the step description and it becomes necessary to keep a larger number of terms in the series.

The first step consists of defining a new step-function $H^{\sim}$ having an average value equal to 0 instead of $\frac{1}{2}$,

$$
H(s)=\frac{1}{2}\left[1+H^{\sim}(s)\right]
$$

With this definition (116), the function $H^{\sim}(s)$ takes the values -1 and +1 for $s<0$ and $s>0$ respectively. Taking account of the basic relation transforming the sinus of a sum into a sum of products of sin and cos functions, an approximation by a trigonometric series is retained. A Fourier expansion of $H^{\sim}$ with a period of $2 L_{S}$ for the reasons mentioned above takes the form

$$
H^{\sim}(s)=\sum_{1}^{\infty} h_{n}^{\sim} \sin \left(\frac{n \pi s}{L_{S}}\right)
$$

with the following coefficients obtained by integration,

$$
h_{n}^{\sim}=\frac{2}{n \pi}\left[1-(-1)^{n}\right]
$$

Inserting (118) and (117) in (116), and using a new index $m$ such as $n=2 m-1$ give

$$
H(s)=\frac{1}{2}+\frac{2}{\pi} \sum_{m=1}^{\infty} \frac{1}{2 m-1} \sin \left(\frac{(2 m-1) \pi s}{L_{S}}\right)
$$

From this trigonometric series for the Heaviside function, we can deduce by direct comparison of Eq. (119) with the Eq. (54) the coefficients $h_{m}$ and wave numbers $\omega_{m}$ introduced in Section 3.2. Keeping for instance only the average value and the first two harmonics of the series (refhvseries), we obtain

$$
H(s)=\frac{1}{2}+\frac{2}{\pi}\left[\sin \left(\frac{\pi s}{L_{S}}\right)+\frac{1}{3} \sin \left(\frac{3 \pi s}{L_{S}}\right)\right]
$$

and it is known from preceding work on microwave instabilities of a bunched beam [20] that retaining indeed only a few harmonics gives an excellent approximation. Fig. 7 gives an illustration of the difference between the $H^{\sim}$ step-function and its representation by the average and first two harmonics. 


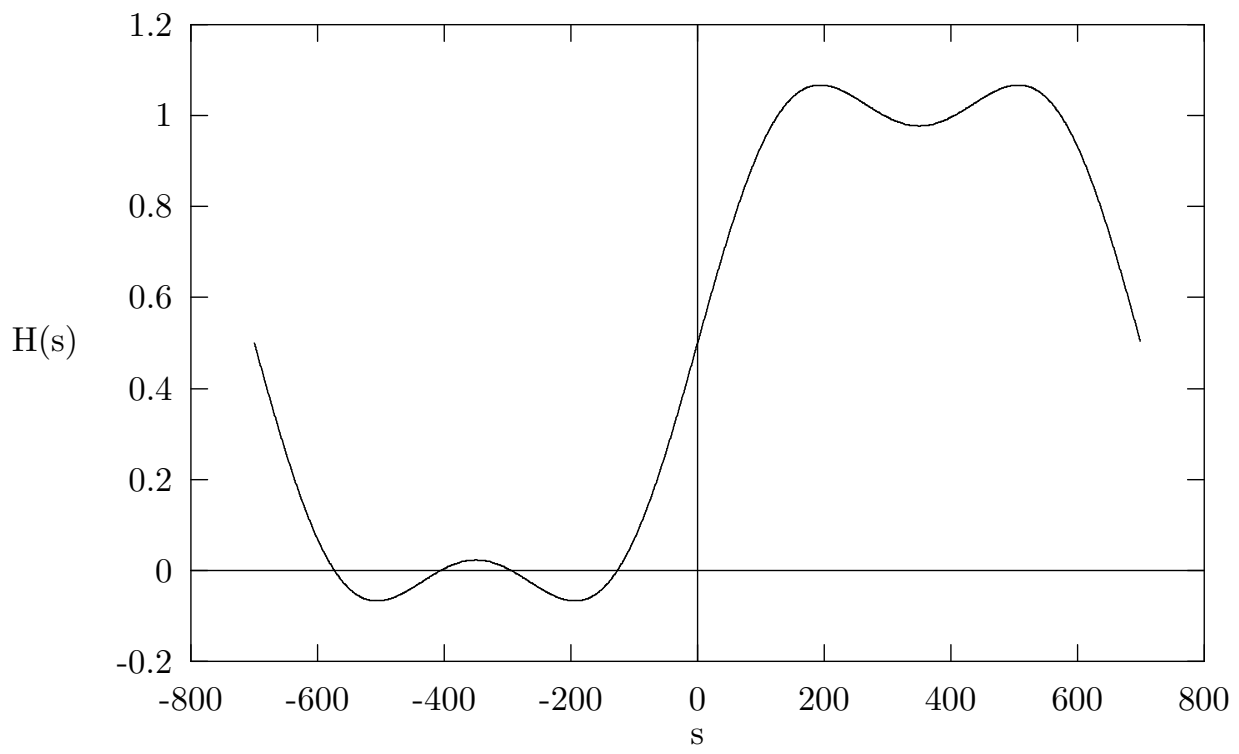

Fig. 7 Fourier Representation of the Heaviside Function

The other property of the Heaviside funtion which is crucial in solving the equation for the zero-order of the perturbation concerns the integral of the product of an arbitrary function $f\left(s^{*}\right)$ by the function $H\left(s^{*}-s_{k}\right)$ (describing a step in $s_{k}$ ), over an interval $[0, s]$, i.e.

$$
J=\int_{0}^{s} f\left(s^{*}\right) H\left(s^{*}-s_{k}\right) d s^{*}
$$

Considering the definition oh $H\left(s^{*}-s_{k}\right)$, we obtain for the integral,

$$
\begin{gathered}
s \leq s_{k} \quad J=0 \\
s>s_{k} \quad J=\int_{0}^{s_{k}} f\left(s^{*}\right) H\left(s^{*}-s_{k}\right) d s^{*}+\int_{s_{k}}^{s} f\left(s^{*}\right) H\left(s^{*}-s_{k}\right) d s^{*}
\end{gathered}
$$

In the first integral of (123) we have $H\left(s^{*}-s_{k}\right)=0$ and in the second $H\left(s^{*}-s_{k}\right)=1$. This simply means

$$
\begin{gathered}
s \leq s_{k} \quad J=0 \\
s>s_{k} \quad J=\int_{s_{k}}^{s} f\left(s^{*}\right) d s^{*}
\end{gathered}
$$

Let us now define another product of functions, termed $J^{*}$

$$
J^{*}=H\left(s-s_{k}\right) \int_{s_{k}}^{s} f\left(s^{*}\right) d s^{*}
$$

assuming that the analytical extension of the function $f\left(s^{*}\right)$ exists for any $s$, in particular for $s \leq s_{k}$. Using the definition of $H$ given in Section 2.2, we can write the following,

$$
\begin{array}{lll}
J^{*}=-H\left(s-s_{k}\right) \int_{s}^{s_{k}} f\left(s^{*}\right) d s^{*} & \text { if } & s \leq s_{k} \\
J^{*}=+H\left(s-s_{k}\right) \int_{s_{k}}^{s} f\left(s^{*}\right) d s^{*} & \text { if } & s>s_{k}
\end{array}
$$


Simply after replacing $H\left(s-s_{k}\right)$ by its value 0 in the interval $s \leq s_{k}$ or 1 in the interval $s>s_{k}$, this soluton for $J^{*}$ is mathematically equivalent to

$$
\begin{gathered}
s \leq s_{k} \quad J^{*}=0 \\
s>s_{k} \quad J^{*}=\int_{s_{k}}^{s} f\left(s^{*}\right) d s^{*}
\end{gathered}
$$

The thing to note is that the integral over the analytical extension of $f\left(s^{*}\right)$ is simply irrelevant, though it exists, and does not contribute to the final result, because it is multiplied by zero. Finally, the simple comparison of the expressions (124) and (127) proves that

$$
J=J^{*}
$$

which is the identity (57) of Section 3.2. 


\section{The partial perturbation expansion method}

In this theory, a non standard perturbation technique is applied in order to preserve the detuning properties of the dynamical system which describes a charged bunch traveling in a linac with wakefields. In order to justify this method we investigate it for the more general case of a linear operator and a linear or nonlinear perturbing function. Since in solving the transverse equation of motion, we only proceed to first order in the perturbation, the theoretical treatment will also be restricted to the same order although we will arrive at a conjecture covering arbitrary orders in the perturbation expansion.

We consider a problem of the kind:

$$
L_{00}(X)+\epsilon L_{01}(X)=\epsilon P(X)
$$

where $L_{00}$ and $L_{01}$ are linear algebraic or differential operators like for instance,

$$
\frac{\partial^{2}}{\partial t^{2}}+\omega^{2}
$$

and $P(X)$ is a linear or nonlinear operator. As it can be seen the linear part itself consists of an unperturbed part $L_{00}$ and a weak contribution $\epsilon L_{01}$. We require that $L_{00}$ and $L_{01}$ are invertable and that the linearized problem

$$
L_{00}(X)+\epsilon L_{01}(X)=0
$$

has a closed form solution for $X$. We now compare the classical straightforward perturbation expansion of this problem to the partial expansion method used in this paper.

- The classical (total) expansion method to first order:

We rewrite (129) as

$$
L_{00}(X)=\epsilon\left[P(X)-L_{01}(X)\right]
$$

and let

$$
X=X_{0}+\epsilon X_{1}+O\left(\epsilon^{2}\right)
$$

To zero and first order in $\epsilon$ we then obtain

$$
\begin{aligned}
& L_{00}\left(X_{0}\right)=0 \Rightarrow X_{0}=L_{00}^{-1}(0) \\
& L_{00}\left(X_{1}\right)=P\left(X_{0}\right)-L_{01}\left(X_{0}\right) \Rightarrow X_{1}=L_{00}^{-1}\left[P\left(X_{0}\right)-L_{01}\left(X_{0}\right)\right]
\end{aligned}
$$

and thus

$$
X=L_{00}^{-1}(0)+\epsilon L_{00}^{-1}\left[P\left(L_{00}^{-1}(0)\right)-L_{01} L_{00}^{-1}(0)\right]+O\left(\epsilon^{2}\right)
$$

- The partial expansion method:

In this case the entire linear operator serves as the unperturbed part of the equation and a perturbation expansion is only applied to $\epsilon P(X)$. In order to provide this we replace $\epsilon$ in the linear operator part by a general parameter $E$ which after performing the expansion on the remaining $\epsilon$ will be reset to $\epsilon$. Hence,

$$
L_{00}(X)+E L_{01}(X)=\epsilon P(X)
$$

Expanding w.r.t. $\epsilon$ we are lead to

$$
X=X_{0}(E)+\epsilon X_{1}(E)+O\left(\epsilon^{2}\right)
$$


and the equations for $X_{0}(E)$ and $X_{1}(E)$ are

$$
\begin{aligned}
& L_{00}\left(X_{0}\right)+E L_{01}\left(X_{0}\right)=0 \\
& L_{00}\left(X_{1}\right)+E L_{01}\left(X_{1}\right)=P\left(X_{0}\right)
\end{aligned}
$$

Since we are interested only in first order solutions we expand $X_{0}$ and $X_{1}$ in this system w.r.t $E$

$$
\begin{aligned}
& X_{0}=X_{00}+E X_{01} \\
& X_{1}=X_{10}+E X_{11}
\end{aligned}
$$

and by comparing like powers in $E$ we find to first order in $E$ :

$$
\begin{aligned}
& X_{0}(E)=L_{00}^{-1}(0)-E L_{00}^{-1} L_{01} L_{00}^{-1}(0)+O\left(E^{2}\right) \\
& X_{1}=L_{00}^{-1} P\left(L_{00}^{-1}(0)\right)+E X_{11}+O\left(E^{2}\right)
\end{aligned}
$$

Then the solution $X$ according to (138) has the form

$$
X=L_{00}^{-1}(0)-E L_{00}^{-1} L_{01} L_{00}^{-1}(0)+\epsilon\left[L_{00}^{-1} P\left(L_{00}^{-1}(0)\right)+E X_{11}\right]+O\left(\epsilon^{2}\right)
$$

If we replace $E=\epsilon$ in Eq. (145) and expand once more to first order in $\epsilon$ then $X$ becomes

$$
X=L_{00}^{-1}(0)+\epsilon\left[L_{00}^{-1} P\left(L_{00}^{-1}(0)\right)-L_{00}^{-1} L_{01} L_{00}^{-1}(0)\right]+O\left(\epsilon^{2}\right)
$$

which agrees with the result found from the classical expansion method (136). This proof has been extended to second order with the equivalent result. Therefore we make the conjecture stated below.

Conjecture: Partial perturbation expansion applied to Eq. (129) to order $N>0$ leads to an expression

$$
\sum_{n=0}^{N} x_{n}(E) \epsilon^{n}
$$

which, for $E=\epsilon$ agrees up to order $N$ with the total expansion of Eq. (129) w.r.t. $\epsilon$.

It is clear however that, after solving the equations (139) and (140), $X_{0}(E)$ as well as $X_{1}(E)$ contain all powers in $E$ and thus the final result $x(\epsilon)$ will be generally represented by a non polynomial function in the perturbation parameter. Another consequence is that the remainder of the partial perturbation expansion after truncation to a given order $N$ differs from the remainder of the total Taylor expansion of same order. 


\section{The logistic map and random number generation}

In the theory of the chaotic dynamics of nonlinear systems [18], the one-dimensional logistic map is defined by the following function

$$
F_{\mu}=\mu x(1-x)
$$

or by the recurrence relation

$$
x_{k+1}=\mu x_{k}\left(1-x_{k}\right)
$$

In both cases $\mu$ is a parameter that has a strong impact on the behaviour of the map. Its fixed points are given by the equation $x^{*}=F_{\mu}\left(x^{*}\right)$. For $0<\mu<1$ the origin $x=0$ is an attracting fixed point, meaning that all initial points sufficiently close to the attractor converge towards zero after the application of successive mappings. For $1<\mu<3$ all points asymptotically reach the fixed point $x^{*}=(\mu-1) / \mu$. Beyond this value $\mu=3$ the single fixed point bifurcates into two points, which subsequently bifurcate into four points, and so on, till the interval of $\mu$ between successive bifurcations decreases and a chaotic set appears.

As a indicator of chaos, the Lyapunov exponent $\lambda$ is often used. It measures the exponential separations of points initially close to each other, as the map is iterated. Let us briefly recall its definition considering two points $x_{0}$ and $x_{0}+\epsilon$ mapped by the function $f: I \rightarrow I, I \in \Re$ and a number $n$ of iterations of this map.

$$
\epsilon \exp n \lambda\left(x_{0}\right)=f^{n}\left(x_{0}+\epsilon\right)-f^{n}\left(x_{0}\right)
$$

Dividing by $\epsilon$ and taking the limit as $\epsilon \rightarrow 0$ shows that the left hand side exponential is equal to the derivative of $f^{n}(x)$ with respect to $x$, at $x=x_{0}$. Taking eventually the limit for $n \rightarrow \infty$ gives the definition of $\lambda\left(x_{0}\right)$,

$$
\lambda\left(x_{0}\right) \equiv \lim _{n \rightarrow \infty} \frac{1}{n} \ln \left|\frac{d f^{n}(x)}{d x}\right|\left(x_{0}\right)
$$

For $\lambda\left(x_{0}\right)>0$ the Lyapunov coefficient represents the exponential stretching of the distance separating initially nearby points, which characterizes the appearance of chaos. For $\lambda\left(x_{0}\right)$ negative, the map has evidently a periodic behaviour.

Coming back to the logistic map (148), the Lyapunov exponent has been calculated as a function of $\mu$ [ref.], in the interval [2.5;4.0]. Though the variation of $\lambda$ is irregular, its values remain negative as long as $\mu$ is smaller than 3.57 approximately. This means that above this value (close to the point of convergence of the sequence of the bifurcations), the map tends toward a chaotic behaviour.

Let us now consider the particular case where $\mu=4$, since the corresponding logistic map is chaotic, given the arguments mentioned above, and the recurrence $x_{n}$ can be written using simple algebraic functions, as shown hereafter. Starting from

$$
x_{n+1}=4 x_{n}\left(1-x_{n}\right)
$$

let us substitute [18] $x_{n}=\sin ^{2} \theta_{n}$ into (152). After some simple algebra it comes out that

$$
\left(\sin \theta_{n+1}\right)^{2}=\left(\sin 2 \theta_{n}\right)^{2}
$$

so as

$$
\theta_{n+1}=2 \theta_{n} \quad \text { and } \quad \theta_{n}=2^{n} \theta_{0}
$$


The relations (153) and (154) prove that the sequence defined by (152) can be rewritten in the form $x_{n}=\left(\sin 2^{n} \theta_{0}\right)^{2}$. Furthermore, since $x_{0}$ is in the interval $[0 ; 1]$, the initial value $\theta_{0}$ can be written as $\beta \pi$ and $\theta_{n}$ as $2^{n} \beta \pi$, with $\beta \in[0 ; 1]$. It is then interesting to write $\beta$ in a binary form like

$$
\beta=\sum_{\nu=1}^{\infty} \frac{b_{\nu}}{2^{\nu}}
$$

Indeed, it can be proven [18] using this particular form (155) that, when the initial point is only specified with finite accuracy, then the digits of its binary representation after a few applications of the map are random. The important consequence for us is that the subsequent applications of the map result in a sequence of random numbers. This is the precise link we need between the chaotic dynamics and the generation of random numbers.

What precedes explains our choice of the function $\left(\sin \theta_{0} 2^{k}\right)^{2}$ in Section 6.1 (Eq.(90)) to provide random numbers. As mentioned there, the term $2^{k}$ can be developed into a series of powers of $\mathrm{k}$, following

$$
2^{k}=\sum_{m=0}^{\infty} \frac{1}{m !}(k \ln 2)^{m}
$$

By extension of the preceding map-analysis results, we claim a priori that the sin-function of the first (for simplicity) non-linear term in $k$ of the series (156) will generate a succession of random numbers $x_{k}^{r}$, according to the equation (91) in Section 6.1. This conjecture remains of course to be verified. For this, we use the autocorrelation function defined as

$$
\begin{aligned}
& g(\tau)=\lim _{T \rightarrow \infty} \frac{1}{T} \int_{-T}^{T} x(t) x(t+\tau) d t \\
& \text { with } \quad x(t)=\sin \left(a t^{2}\right)
\end{aligned}
$$

In case of perfect randomness, the result of the integral (157) is a Dirac-funtion at the origin (correlation when $\tau \rightarrow 0$ ), with vanishing values elsewhere (no correlation as soon as $\tau \neq 0$ ). With the particular function retained, the integral (157) writes as follows

$$
g(\tau)=\int_{-T}^{T} \sin \left(a t^{2}\right) \sin \left((a(t+\tau))^{2}\right) d t
$$

and can be expressed in an explicit form [16], which is given hereafter for an arbitrary interval $T$ and coefficient $a$

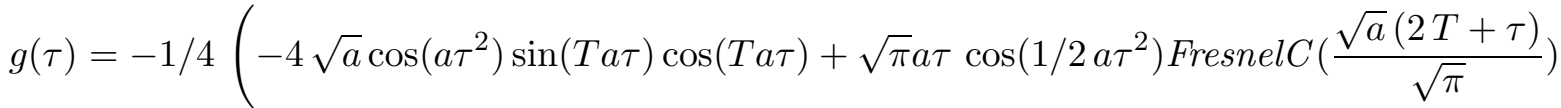

$$
\begin{aligned}
& -\sqrt{\pi} a \tau \sin \left(1 / 2 a \tau^{2}\right) \text { Fresnel }\left(\frac{\sqrt{a}(2 T+\tau)}{\sqrt{\pi}}\right)-\sqrt{\pi} a \tau \cos \left(1 / 2 a \tau^{2}\right) \operatorname{Fresnel} C\left(\frac{\sqrt{a}(-2 T+\tau)}{\sqrt{\pi}}\right) \\
& \left.+\sqrt{\pi} a \tau \sin \left(1 / 2 a \tau^{2}\right) \text { FresnelS }\left(\frac{\sqrt{a}(-2 T+\tau)}{\sqrt{\pi}}\right)\right) T^{-1} a^{-3 / 2} \tau^{-1}
\end{aligned}
$$

Let us now estimate the function $g(\tau)$ for a particular value $a=1$ and a finite period arbitrarily large, i.e. $T=100$. The corresponding expression, deduced from (160), is 


$$
\begin{aligned}
& g(\tau, T=100, a=1)=\frac{1}{400}\left(4 \cos \left(\tau^{2}\right) \sin (100 \tau) \cos (100 \tau)-\sqrt{\pi} \tau \cos \left(1 / 2 \tau^{2}\right) \text { FresnelC }\left(\frac{200+\tau}{\sqrt{\pi}}\right)\right. \\
& +\sqrt{\pi} \tau \sin \left(1 / 2 \tau^{2}\right) \text { FresnelS }\left(\frac{200+\tau}{\sqrt{\pi}}\right)+\sqrt{\pi} \tau \cos \left(1 / 2 \tau^{2}\right) \text { FresnelC }\left(\frac{-200+\tau}{\sqrt{\pi}}\right) \\
& \left.-\sqrt{\pi} \tau \sin \left(1 / 2 \tau^{2}\right) \text { Fresnel }\left(\frac{-200+\tau}{\sqrt{\pi}}\right)\right) \tau^{-1}
\end{aligned}
$$

Fig. 8 shows that the autocorrelation function (161) obtained by numerical integration exhibits the properties required for randomness. The residual oscillations (called Gibbs phenomenon) on either side of the sharp peak, located at $\tau=0$ as expected, are related to the fact that the interval $[-T, T]$ of integration is necessarily finite. When $T$ increases, these oscillations shrink more and more toward the origin and eventually leave a Dirac-function. In addition, the ratio of the peak- to the tail-amplitudes is larger than an order of magnitude in this particular case and also increases with the value of $T$. Hence, it is logically possible to conclude that the autocorrelation defined by (157) is minimal for $\sin \left(a t^{2}\right)$, except when $\tau \rightarrow 0$. Hence, by definition, the sequence of numbers defined by the Eq. (91) in Section 6.1 is actually random as desired. The coefficient $a$ initiates a particular sequence of random numbers and a small variation of $a$ induces a totally different sequence since the behaviour is chaotic. In other words, $a$ plays the role of the "seed" in a numerical iterative algorithm that generates random numbers.

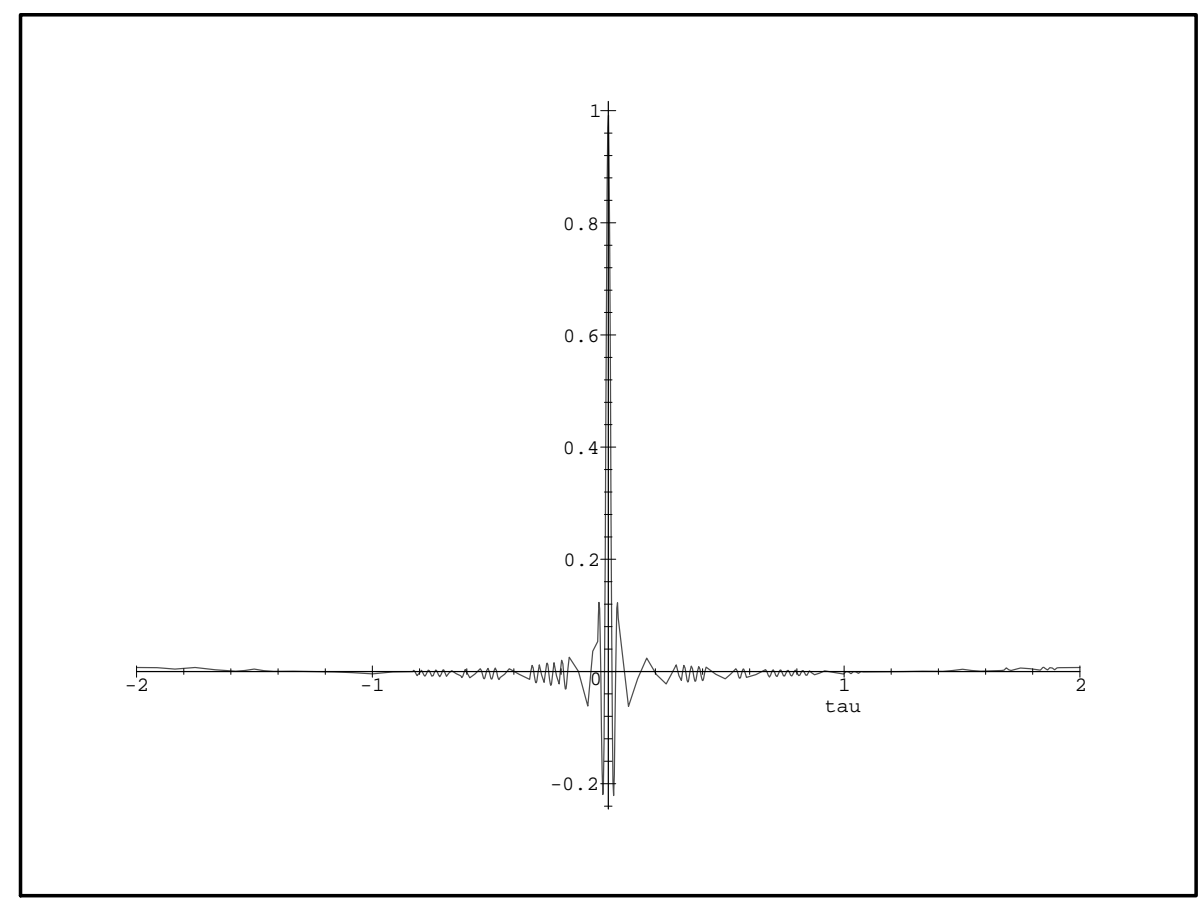

Fig. 8 Autocorrelation function for the algebraic form sin $a t^{2}$ 